\title{
VOLTERRA CALCULUS, LOCAL EQUIVARIANT FAMILY INDEX THEOREM AND EQUIVARIANT ETA FORMS*
}

\author{
YONG WANG ${ }^{\dagger}$
}

\begin{abstract}
In this paper, we give proofs of the family index formula and the equivariant family index formula by the Greiner's approach to heat kernel asymptotics. We compute equivariant family JLO characters. We also define the equivariant eta form and give a proof of its regularity.
\end{abstract}

Key words. Heat equation methods, family index formula, equivariant family index formula, equivariant family JLO characters, equivariant eta form.

AMS subject classifications. 58J20.

1. Introduction. The first success of proving the Atiyah-Singer index theorem directly by heat kernel method was achieved by Patodi $[\mathrm{Pa}]$, who carried out the "fantastic cancellation" (cf. [MS]) for the Laplace operators and who for the first time proved a local version of the Gauss-Bonnet-Chern theorem. After that several different direct heat kernel proofs of the Atiyah-Singer index theorem for Dirac operators appeared independently: Bismut [Bi1], Getzler [Ge1], [Ge2] and $\mathrm{Yu}[\mathrm{Yu}]$, Ponge [Po1]. All the proofs have their own advantages. Motivated by the problem of generalizing the heat kernel proofs of the index theorem to prove a local index theorem for families of elliptic operators, Quillen $[\mathrm{Qu}]$ introduced the concept of superconnections, which was developed by Bismut to give a heat kernel representation for the Chern character of families of first order elliptic operators. Then using his probabilitistic method, Bismut [Bi2] obtained a proof of the local index theorem for families of Dirac operators. In [BV1],[Do1], they gave two different proofs of local index theorem for families of Dirac operators. Using the method of $\mathrm{Yu}$, Zhang gave another proof of the local index theorem for families of Dirac operators in [Zh1]. The first purpose of this paper is to give another proof of the local index theorem for families of Dirac operators by the Ponge's method in [Po1].

The Atiyah-Bott-Segal-Singer index formula is a generalization of the AtiyahSinger index theorem to the case with group actions. In [BV2], Berline and Vergne gave a heat kernel proof of the Atiyah-Bott-Segal-Singer index formula. In [LYZ], Lafferty, Yu and Zhang presented a very simple and direct geometric proof for equivariant index of the Dirac operator. In [PW], Ponge and Wang gave a different proof of the equivariant index formula by the Greiner's approach of the heat kernel asymptotics. In [LM], in order to prove family rigidity theorems, Liu and Ma proved the equivariant family index formula. The second purpose of this paper is to give another proof of the local equivarint index theorem for families of Dirac operators by the Greiner's approach of the heat kernel asymptotics.

It is known, due to Connes [Co] and an equivalent but convenient version due to Jaffe, Lesniewski and Osterwalder, also known as a JLO formula [JLO], that the Chern character of a $\theta$-summable Fredholm module $(H, D)$ over a unital $C^{*}$-algebra $A$, takes value in the entire cyclic cohomology of $A$. JLO characters were computed in [CM1] and $[\mathrm{BlF}]$. An explicit formula of an equivariant JLO character, associated to the invariant Dirac operator, in the presence of a countable discrete group action on

\footnotetext{
*Received April 19, 2013; accepted for publication April 24, 2015.

†School of Mathematics and Statistics, Northeast Normal University, Changchun Jilin, 130024, China (wangy581@nenu.edu.cn).
} 
a smooth compact spin Riemannian manifold, was given by Azmi, [Az1]. Moreover, in [Az1] it was shown that this equivariant cocycle is an element of the delocalized cohomology, and it can be paired with an equivariant $K$-theory idempotent. In the case when $G$ is a compact Lie group, Chern and $\mathrm{Hu}[\mathrm{CH}]$ gave an explicit formula of an equivariant Chern-Connes character, associated to a G-equivariant $\theta$-summable Fredholm module. In [PW], Ponge and Wang computed equivariant Chern-Connes characters by the Greiner's approach of the heat kernel asymptotics.

On the other hand, Wu [Wu] constructed a bivariant Chern-Connes character for (a special class of) $\theta$ summable modules, by incorporating the JLO formula and the superconnectiom formalism of Quillen. Wu's bivariant character takes values in the bivariant cyclic theory described by Lott [Lo], who constructed it as a combination of entire cyclic (co)homology and noncommutative de Rham homology of graded differential algebra. Then by adopting Wu's method and employing Bismut's superconnection together with the canonical order calculus developed by Simon in [CFKB], Azmi [Az2] expressed the local formula for families in terms of differential forms on the base and the Chern roots of the fibration. In [Zh2], Zhang announced a different method to compute the family JLO characters by developing the family version of the method in $[\mathrm{CH}]$ and $[\mathrm{Fe}]$. In the third part of this paper, we give details of the announcement in [Zh2] and compute the equivariant family JLO characters.

In [APS], Atiyah, Patodi and Singer proved the Atiyah-Patodi-Singer index theorem for manifolds with boundary and they introduced the eta invariants. Bismut and Freed gave a simple proof of the regularity of eta invariants in $[\mathrm{BiF}]$. In [Po2], Ponge gave another proof of the regularity of eta invariants using the method in [Po1]. Bismut and Cheeger generalized the Atiyah-Patodi-Singer index theorem to the family case in $[\mathrm{BC} 1,2]$. They used the eta form for families of Dirac operators. The regularity of the eta form was proved by the probabilistic method in [BiGS]. Donnelly generalized the Atiyah-Patodi-Singer index theorem to the equivariant case and introduced the equivariant eta invariant in [Do2]. Zhang proved the regularity of the equivariant eta invariant by the Clifford asymptotics in [Zh3]. In this paper, we firstly prove the regularity of the equivariant eta invariant by a similar method in $[\mathrm{PW}]$ and by introducing the Grassmann variable. Then we define the equivariant eta form and prove its regularity.

Using the approach of Ponge and Ponge-Wang to give new proofs of the equivariant family index theorem and the regularity of equivariant eta forms has two advantages. One is using the Volterra pseudodifferential calculus to get the heat kernel asymtotic expansion instead of heat equation discussions as in [Yu], [Zh1], [BGV, Chapter 2]. In [LYZ] and [Zh3], for proving the equivariant local index theorem and the regularity of the equivariant eta invariants, transformed formulas between normal and tubular coordinates are needed. In [LM], Liu and Ma used the finite propagation speed method in [Bi3] to prove the equivariant family index theorem. The other advantage is that the transformed formulas between normal and tubular coordinates are the consequence of a standard change of variable formula for pseudodifferential symbols.

This paper is organized as follows: In Section 2, we give another proof of the local index theorem for families of Dirac operators by the Ponge's method. In Section 3, we give another proof of the local equivarint index theorem for families of Dirac operators by the Greiner's approach of the heat kernel asymptotics. In Section 4, we compute the equivariant family JLO characters. In Section 5 , we define the equivariant eta form and prove its regularity. 


\section{The local index theorem for families of Dirac operators.}

2.1. The Greiner's approach of heat kernel asymptotics with form coefficients. In [Gr],[BGS], Greiner and Beals-Greiner-Stanton defined Volterra pseudodifferential operators and gave the heat kernel asymptotics by Volterra pseudodifferential calculus. A good summary on the Greiner's approach of the heat kernel asymptotics was given in [Po1]. In [Po1], Ponge gave a proof of the local index theorem by the Greiner's approach of the heat kernel asymptotics. In this section, we shall extend Volterra pseudodifferential calculus to the case with form coeffcients, i.e. the family case. A local family index theorem was proved originally by Bismut in [Bi2]. A superconnection due to Bismut played an important role in the proof of the local family index theorem.

Let us recall the definition of superconnection due to Bismut. Let $M$ be an $n+\bar{q}$ dimensional compact connected manifold and $B$ be a $\bar{q}$ dimensional compact connected manifold. We assume that $\pi: M \rightarrow B$ is a submersion of $M$ onto $B$, which defines a fibration of $M$ with fibre $Z$. For $y \in B, \pi^{-1}(y)$ is then a submanifolds $M_{y}$ of $M$. Denote by $T Z$ the $n$-dimensional vector bundle on $M$ whose fibre $T_{x} M_{\pi x}$ is the tangent space at $x$ to the fibre $M_{\pi(x)}$. We assume that $M$ and $B$ are oriented. We take a smooth horizontal subbundle $T^{H} M$ of $T M$. Vector fields $X \in \Gamma(B, T B)$ will be identified with their horizontal lifts $X \in \Gamma\left(M, T^{H} M\right)$. Moreover $T_{x}^{H} M$ is isomorphic to $T_{\pi(x)} B$ via $\pi_{*}$. We take a Riemannian metric on $B$ and then lift the Euclidean scalar product $g_{B}$ of $T B$ to $T^{H} M$. We further assume that $T Z$ is endowed with a scalar product $g_{Z}$. Thus we can introduce on $T M$ a new scalar product $g_{B} \oplus g_{Z}$, and denote by $\nabla^{L}$ the Levi-Civita connection on $T M$ with respect to this metric. Set $\nabla^{B}$ denote the Levi-Civita connection on $T B$ and we still denote by $\nabla^{B}$ the pullback connection on $T^{H} M$. Let $\nabla^{Z}=P_{Z}\left(\nabla^{L}\right)$ where $P_{Z}$ denotes the orthogonal projection to $T Z$. Set $\nabla^{\oplus}=\nabla^{B} \oplus \nabla^{Z}$ and $S=\nabla^{L}-\nabla^{\oplus}$ and $T$ be the torsion tensor of $\nabla^{\oplus}$. Denote by $S O(T Z)$ the $S O(n)$ bundle of oriented orthonormal frames in $T Z$. Now we assume that bundle $T Z$ is spin. Denote by $S(T Z)$ the associated spinor bundle and $\nabla^{Z}$ can be lifted to a connection on $S(T Z)$. Let $D$ be the Dirac operator in the tangent direction defined by $D=\sum_{i=1}^{n} c\left(e_{i}^{*}\right) \nabla_{e_{i}}^{S(T Z)}$ where $\nabla^{S(T Z)}$ is a spin connection on $S(T Z)$. Set $E$ be the vector bundle $\pi^{*}\left(\wedge T^{*} B\right) \otimes S(T Z)$. This bundle carries a natural action $m_{0}$ of the degenerate Clifford module denoted as $C l_{0}(M)$. The Clifford action of a horizontal cotangent vector $\alpha \in \Gamma\left(M, T_{H}^{*} M\right)$ is given by the exterior multiplication $m_{0}(\alpha)=\varepsilon(\alpha)$ acting on the first factor $\bigwedge T_{H}^{*} M$ in $E$, while the Clifford action of a vertical cotangent vector simply is its Clifford action on $S(T Z)$. Define the connection by $([\mathrm{BGV}])$

$$
\begin{gathered}
\nabla^{E, \oplus}:=\pi^{*} \nabla^{B} \otimes 1+1 \otimes \nabla^{S}, \\
\omega(X)(Y, Z):=g\left(\nabla_{X}^{L} Y, Z\right)-g\left(\nabla_{X}^{\oplus} Y, Z\right), \\
\nabla_{X}^{E, 0}:=\nabla_{X}^{E, \oplus}+\frac{1}{2} m_{0}(\omega(X)),
\end{gathered}
$$

for $X, Y, Z \in \Gamma(M, T M)$. Then the Bismut connection acting on $\Gamma\left(M, \wedge\left(\pi^{*} T^{*} B\right) \otimes\right.$ $S(T Z))$ is defined by

$$
\mathcal{B}=\sum_{i=1}^{n} c\left(e_{i}^{*}\right) \nabla_{e_{i}}^{E, 0}+\sum_{j=1}^{\bar{q}} c\left(f_{j}^{*}\right) \nabla_{f_{j}}^{E, 0}
$$


Then by Proposition 10.15 in $[\mathrm{BGV}], \mathcal{B}=D+\mathrm{A}_{[+]}$, where $\mathrm{A}_{[+]}$is an operator with coefficients in $\Omega_{\geq 1}(B)$. By Theorem 10.17 in [BGV], we have

$$
\mathrm{F}=\mathcal{B}^{2}=-\sum_{i=1}^{n}\left(\nabla_{e_{i}}^{E, 0}\right)^{2}+\sum_{i=1}^{n} \nabla_{\nabla_{e_{i}}^{T Z} e_{i}}^{E, 0}+\frac{1}{4} r=D^{2}+F_{[+]},
$$

where $r$ is the scalar curvature of fibres. Let $\triangle_{k}$ be the $k$-simplex defined by

$$
\left\{\left(\sigma_{0}, \cdots, \sigma_{k}\right) \mid \sigma_{0}+\cdots \sigma_{k}=1,0 \leq \sigma_{j} \leq 1\right\} .
$$

For a fixed $t>0$, define the operator $e^{-t F}$ by

$$
e^{-t F}=e^{-t D^{2}}+\sum_{k>0}(-t)^{k} I_{k}
$$

where

$$
I_{k}=\int_{\triangle_{k}} e^{-\sigma_{0} t D^{2}} F_{[+]} e^{-\sigma_{1} t D^{2}} F_{[+]} \cdots e^{-\sigma_{k-1} t D^{2}} F_{[+]} e^{-\sigma_{k} t D^{2}} d \sigma
$$

and where the sum is finite (see [BGV, p. 312]). Then

$$
\left(\frac{\partial}{\partial t}+F\right) e^{-t F}=0, \quad F e^{-t F}=e^{-t F} F .
$$

In the following, we formulate the Greiner's approach of heat kernel asymptotics with coefficients in $\wedge T_{z}^{*} B$ as in [Gr] and [BeGS]. Let $Q_{0}$ be given by

$$
\left(Q_{0} u\right)(x, s)=\int_{0}^{\infty} e^{-s F}[u(x, t-s)] d t, \quad u \in \Gamma_{c}\left(M_{z} \times \mathbb{R}, S\left(T M_{z}\right)\right) \otimes \wedge T_{z}^{*} B .
$$

The operator $Q_{0}$ maps continuously from $u$ to $D^{\prime}\left(M_{z} \times \mathbb{R}, S\left(T M_{z}\right)\right) \otimes \wedge T_{z}^{*} B$ which is the dual space of $\Gamma_{c}\left(M_{z} \times \mathbb{R}, S\left(T M_{z}\right)\right) \otimes \wedge T_{z}^{*} B$. By (2.8), we have

$$
\left(F+\frac{\partial}{\partial t}\right) Q_{0} u=Q_{0}\left(F+\frac{\partial}{\partial t}\right) u=u, \quad u \in \Gamma_{c}\left(M_{z} \times \mathbb{R}, S\left(T M_{z}\right)\right) \otimes \wedge T_{z}^{*} B,
$$

We define the operator

$$
Q=\left(F+\frac{\partial}{\partial t}\right)^{-1}=\left(D^{2}+\frac{\partial}{\partial t}\right)^{-1}+\sum_{k>0}(-1)^{k}\left(D^{2}+\frac{\partial}{\partial t}\right)^{-1}\left[F_{[+]}\left(D^{2}+\frac{\partial}{\partial t}\right)^{-1}\right]^{k}
$$

where $\left(D^{2}+\frac{\partial}{\partial t}\right)^{-1}$ is the Volterra inverse of $D^{2}+\frac{\partial}{\partial t}$ as in [BeGS]. Then

$$
\left(F+\frac{\partial}{\partial t}\right) Q=I-R_{1} ; \quad Q\left(F+\frac{\partial}{\partial t}\right)=1-R_{2},
$$

where $R_{1}, R_{2}$ are smoothing operators. Let $K_{Q_{0}}(x, y, t-s)$ be the distribution kernel of $Q_{0}$. That is

$$
\left(Q_{0} u\right)(x, t)=\int_{M_{z} \times \mathbb{R}} K_{Q_{0}}(x, y, t-s) u(y, s) d y d s,
$$

and let $k_{t}(x, y)$ be the heat kernel of $e^{-t F}$. Similar to the discussions in [BeGS p.363], we get

$$
K_{Q_{0}}(x, y, t)= \begin{cases}k_{t}(x, y) & \text { when } t>0 \\ 0 & \text { when } t<0\end{cases}
$$


Definition 2.1. The operator $P$ is called a Volterra $\Psi D O$ if (i) $P$ has the Volterra property, i.e. it has a distribution kernel of the form $K_{P}(x, y, t-s)$ where $K_{P}(x, y, t)$ vanishes on the region $t<0$.

(ii) The heat operator $P+\frac{\partial}{\partial t}$ is parabolically homogeneous, i.e. the homogeneity with respect to the dilations of $\mathbb{R}^{n} \times \mathbb{R}^{1}$ is given by

$$
\lambda \cdot(\xi, \tau)=\left(\lambda \xi, \lambda^{2} \tau\right), \quad(\xi, \tau) \in \mathbb{R}^{n} \times \mathbb{R}^{1}, \quad \lambda \neq 0 .
$$

In the sequel for $g \in \mathcal{S}\left(\mathbb{R}^{n+1}\right)$ and $\lambda \neq 0$, we let $g_{\lambda}$ be the tempered distribution defined by

$$
\left\langle g_{\lambda}(\xi, \tau), u(\xi, \tau)\right\rangle=|\lambda|^{-(n+2)}\left\langle g(\xi, \tau), u\left(\lambda^{-1} \xi, \lambda^{-2} \tau\right)\right\rangle, \quad u \in \mathcal{S}\left(\mathbb{R}^{n+1}\right) .
$$

Definition 2.2. A distribution $g \in \mathcal{S}\left(\mathbb{R}^{n+1}\right)$ is parabolic homogeneous of degree $m$, where $m \in \mathbb{Z}$, if for any $\lambda \neq 0$, we have $g_{\lambda}=\lambda^{m} g$.

Let $\mathbb{C}_{-}$denote the complex halfplane $\{\operatorname{Im} \tau<0\}$ with closure $\overline{\mathbb{C}_{-}}$. Then:

Lemma 2.3 ([BeGS, Prop. 1.9]). Let $q(\xi, \tau) \in C^{\infty}\left(\left(\mathbb{R}^{n} \times \mathbb{R}\right) \backslash 0\right) \otimes \wedge T_{z}^{*} B$ be a parabolic homogeneous symbol of degree $m$ such that:

(i) $q$ extends to a continuous function on $\left(\mathbb{R}^{n} \times \overline{\mathbb{C}_{-}}\right) \backslash 0$ in such way to be holomorphic in the last variable when the latter is restricted to $\mathbb{C}_{-}$.

Then there is a unique $g \in \mathcal{S}\left(\mathbb{R}^{n+1}\right) \otimes \wedge T_{z}^{*} B$ agreeing with $q$ on $\mathbb{R}^{n+1} \backslash 0$ so that:

(ii) $g$ is homogeneous of degree $m$;

(iii) The inverse Fourier transform $\check{g}(x, t)$ vanishes for $t<0$.

Let $U$ be an open subset of $\mathbb{R}^{n}$. We define Volterra symbols and Volterra $\Psi D O$ s on $U \times \mathbb{R}^{n+1} / 0$ as follows.

Definition 2.4. The set $S_{V}^{m}\left(U \times \mathbb{R}^{n+1}\right) \otimes \wedge T_{z}^{*} B, m \in \mathbb{Z}$, consists of smooth functions $q(x, \xi, \tau)$ on $U \times \mathbb{R}^{n} \times \mathbb{R}$ with an asymptotic expansion $q \sim \sum_{j \geq 0} q_{m-j}$, where:

- $q_{l} \in C^{\infty}\left(U \times\left[\left(\mathbb{R}^{n} \times \mathbb{R}\right) \backslash 0\right]\right) \otimes \wedge T_{z}^{*} B$ is a homogeneous Volterra symbol of degree $l$, i.e. $q_{l}$ is parabolic homogeneous of degree $l$ and satisfies the property (i) in Lemma 2.3 with respect to the last $n+1$ variables;

- The sign $\sim$ means that, for any integer $N$ and any compact $K \subset U$, there is a constant $C_{N K \alpha \beta k}>0$ such that for $x \in K$ and for $|\xi|+|\tau|^{\frac{1}{2}}>1$ we have

$$
\left\|\partial_{x}^{\alpha} \partial_{\xi}^{\beta} \partial_{\tau}^{k}\left(q-\sum_{j<N} q_{m-j}\right)(x, \xi, \tau)\right\| \leq C_{N K \alpha \beta k}\left(|\xi|+|\tau|^{\frac{1}{2}}\right)^{m-N-|\beta|-2 k} .
$$

For $q=\sum_{l} q_{l} \omega^{l}$ where $q_{l} \in S_{V}^{m}\left(U \times \mathbb{R}^{n+1}\right)$ and $\omega^{l} \in \wedge^{l} T_{z}^{*} B$, we define $\|q\|=$ $\sum_{l}\left|q_{l}\right|\left\|\omega^{l}\right\|$ and $\left\|\omega^{l}\right\|$ is the norm of $\omega^{l}$ in $\left(\wedge^{l} T_{z}^{*} B, g_{z}^{T B}\right)$.

Definition 2.5. The set $\Psi_{V}^{m}\left(U \times \mathbb{R}, \wedge T_{z}^{*} B\right), m \in \mathbb{Z}$, consists of continuous operators $Q$ from $C_{c}^{\infty}\left(U_{x} \times \mathbb{R}_{t}, \wedge T_{z}^{*} B\right)$ to $C^{\infty}\left(U_{x} \times \mathbb{R}_{t}, \wedge T_{z}^{*} B\right)$ such that:

(i) $Q$ has the Volterra property;

(ii) $Q=q\left(x, D_{x}, D_{t}\right)+R$ for some symbol $q$ in $S_{V}^{m}\left(U \times \mathbb{R}, \wedge T_{z}^{*} B\right)$ and some smoothing operator $R$. 
In the sequel if $Q$ is a Volterra $\Psi D O$, we let $K_{Q}(x, y, t-s)$ denote its distribution kernel, so that the distribution $K_{Q}(x, y, t)$ vanishes for $t<0$.

Definition 2.6. Let $q_{m}(x, \xi, \tau) \in C^{\infty}\left(U \times\left(\mathbb{R}^{n+1} \backslash 0\right)\right) \otimes \wedge T_{z}^{*} B$ be a homogeneous Volterra symbol of order $m$ and let $g_{m} \in C^{\infty}(U) \otimes \mathcal{S}^{\prime}\left(\mathbb{R}^{n+1}\right) \otimes \wedge T_{z}^{*} B$ denote its unique homogeneous extension given by Lemma 2.3. Then:

- $\breve{q}_{m}(x, y, t)$ is the inverse Fourier transform of $g_{m}(x, \xi, \tau)$ in the last $n+1$ variables; - $q_{m}\left(x, D_{x}, D_{t}\right)$ is the operator with kernel $\breve{q}_{m}(x, y-x, t)$.

The composition of ordinary Volterra symbols naturally extends to a composition of Volterra symbols with form coefficients. Let $\mathbf{Q}$ and $\mathbf{Q}^{\prime}$ be in $\Psi_{V}^{m_{1}}\left(U \times \mathbb{R}, \wedge^{j} T_{z}^{*} B\right)$ and $\Psi_{V}^{m_{2}}\left(U \times \mathbb{R}, \wedge^{l} T_{z}^{*} B\right)$ with the symbols $\mathbf{q}$ and $\mathbf{q}^{\prime}$. Let the composition of $\mathbf{Q}$ and $\mathbf{Q}^{\prime}$ have the symbol $\mathbf{q} \widetilde{\mathbf{o}} \mathbf{q}^{\prime}$, then

$$
\mathbf{q} \widetilde{\mathbf{q}^{\prime}}=\omega_{1} \wedge \omega_{2} \otimes q \circ q^{\prime}
$$

where $\mathbf{q}=\omega_{1} q, \mathbf{q}^{\prime}=\omega_{2} q^{\prime}$ and $q \circ q^{\prime}$ is the ordinary composition of symbols corresponding to the Volterra $\Psi D O$ algebra multiplication ([Gr, BeGs]). Thus we have

$$
\Psi_{V}^{m_{1}}\left(U \times \mathbb{R}, \wedge^{j} T_{z}^{*} B\right) \times \Psi_{V}^{m_{2}}\left(U \times \mathbb{R}, \wedge^{l} T_{z}^{*} B\right) \rightarrow \Psi_{V}^{m_{1}+m_{2}}\left(U \times \mathbb{R}, \wedge^{j+l} T_{z}^{*} B\right) .
$$

Proposition 2.7. The following properties hold.

1) Composition. Let $Q_{j} \in \Psi_{V}^{m_{j}}(U \times \mathbb{R}) \otimes \wedge T_{z}^{*} B, j=1,2$ have symbol $q_{j}$ and suppose that $Q_{1}$ or $Q_{2}$ is properly supported (see [Ta, p. 43]). Then $Q_{1} Q_{2}$ is a Volterra $\Psi D O$ of order $m_{1}+m_{2}$ with symbol $q_{1} \widetilde{\circ} q_{2} \sim \sum \frac{1}{\alpha !} \partial_{\xi}^{\alpha} q_{1} D_{x}^{\alpha} q_{2}$.

2) Parametrices. Let $Q=Q^{m}+Q^{<m}$ where $Q^{m}$ is in $\Psi_{V}^{m}(U \times \mathbb{R})$ (an order $m$ Volterra $\Psi D O$ without form coefficients in $\left.\wedge T_{z}^{*} B\right)$ and $Q^{<m}$ is in $\Psi_{V}^{<m}(U \times \mathbb{R}) \otimes \wedge T_{z}^{*} B$. We assume that there is an operator $P$ in $\Psi_{V}^{-m}(U \times \mathbb{R})$ such that

$$
Q^{m} P=1-R_{1}, \quad P Q^{m}=1-R_{2}
$$

where $R_{1}, R_{2}$ are smoothing operators in $\Psi_{V}^{-\infty}(U \times \mathbb{R})$. let

$$
\widetilde{Q}=P+\sum_{k>0}(-1)^{k} P\left[Q^{<m} P\right]^{k}
$$

then

$$
Q \widetilde{Q}=1-\widetilde{R_{1}}, \quad \widetilde{Q} Q=1-\widetilde{R_{2}}
$$

where $\widetilde{R}_{1}, \widetilde{R}_{2}$ are smoothing operators in $\Psi_{V}^{-\infty}(U \times \mathbb{R}) \otimes \wedge T_{z}^{*} B$.

Proof. The claim 1) comes from (2.28) and 1) of Proposition 1 in [Po1]. By (2.30) and (2.31) and direct computations, we get (2.32).

By (2.11) and the fact that $\left(D^{2}+\partial_{t}\right)^{-1}$ is a Volterra $\Psi D O$ of order -2 and that $F_{[+]}$is a first order Volterra $\Psi D O$, we get

Proposition 2.8. The differential operator $F+\partial_{t}$ is invertible and its inverse $\left(F+\partial_{t}\right)^{-1}$ is a Volterra $\Psi$ DO of order -2 .

Let $Q \in \Psi_{V}^{m}\left(U \times \mathbb{R}, \wedge T_{z}^{*} B\right)$ have symbol $q \sim \sum q_{m-j}$ and $q_{m-j}=\sum_{s=1}^{2^{\bar{q}}} q_{m-j, s} \omega^{s}$ where $q_{m-j, s} \in S^{m-j}(U \times \mathbb{R})$ and $\omega^{s} \in \wedge^{s} T^{*} B$. We define the inverse Fourier transform of $q_{m-j}$ by $\check{q}_{m-j}=\sum_{s=1}^{2^{\bar{q}}} \check{q}_{m-j, s} \omega^{s}$. Then we have 
Lemma 2.9 (Compare with Lemma 2 in [Po1]). Let $Q \in \Psi_{V}^{m}\left(U \times \mathbb{R}, \wedge T_{z}^{*} B\right)$, we have in local coordinates

$$
K_{Q}(x, x, t) \sim t^{-\left(\frac{n}{2}+\left[\frac{m}{2}\right]+1\right)} \sum_{l \geq 0} t^{l} \check{q}_{2\left[\frac{m}{2}\right]-2 l}(x, 0,1) .
$$

Proof. Let $Q=\sum_{r=1}^{2^{\bar{q}}} Q_{r} \omega_{r}$ where $Q_{r} \in \Psi_{V}^{m}(U \times \mathbb{R})$ and $\omega_{r} \in \wedge^{r} T^{*} B$. We note that the leading symbol of $Q_{r}$ is probably zero. We set that the symbol $q^{[r]}$ of $Q_{r}$ has an asymptotic expansion $q^{[r]} \sim \sum_{j \geq 0} q_{m-j}^{[r]}$. By Lemma 2 in [Po1], we have

$$
K_{Q_{r}}(x, x, t) \sim t^{-\left(\frac{n}{2}+\left[\frac{m}{2}\right]+1\right)} \sum_{l \geq 0} t^{l} \breve{q}_{2\left[\frac{m}{2}\right]-2 l}^{[r]}(x, 0,1) .
$$

By the equality

$$
K_{Q}(x, x, t)=\sum_{r=1}^{2^{\bar{q}}} K_{Q_{r}}(x, x, t) \omega_{r}
$$

and the definition of the inverse Fourier transform of $q_{m-j}$, we get (2.33).

By (2.24) and Proposition 2.8 and Lemma 2.9, we get

Theorem 2.10 (Compare with Theorem 1.6.1 in [Gr]). In

$$
C^{\infty}\left(M_{z}, \operatorname{End}\left(S\left(T\left(M_{z}\right)\right)\right)\right) \otimes \wedge T_{z}^{*} B
$$

we have

$$
k_{t}(x, x) \sim t^{-\frac{n}{2}} \sum_{l \geq 0} t^{l} a_{l}(F)(x), t \rightarrow 0^{+}, \quad a_{l}(F)(x)=\check{q}_{-2-2 l}(x, 0,1),
$$

where $q_{-2-2 l}(x, \xi, \tau)$ is the $-2 l-2$ order symbol of $\left(\partial_{t}+F\right)^{-1}$ and the second equality in (2.36) holds in local coordinates.

By the same reason with Lemma 2.9 and Proposition 2 in [Po1], we have

Proposition 2.11 (Compare with Proposition 2 in [Po1]). Let $P$ : $C^{\infty}\left(M_{z}, S\left(T M_{z}\right)\right) \rightarrow C^{\infty}\left(M_{z}, S\left(T M_{z}\right)\right)$ be a differential operator of order $m$ and let $h_{t}(x, y)$ denote the distribution kernel of $P e^{-t F}$. Then in $C^{\infty}\left(M_{z}, \operatorname{End}\left(S\left(T M_{z}\right)\right)\right) \otimes$ $\wedge T_{z}^{*} B$, we have

$$
h_{t}(x, x) \sim t^{-\left(\left[\frac{m}{2}\right]+\frac{n}{2}\right)} \sum_{l \geq 0} t^{l} b_{l}(F)(x), t \rightarrow 0^{+}, \quad b_{l}(x)=\check{q}_{2\left[\frac{m}{2}\right]-2-2 l}(x, 0,1),
$$

where $q_{2\left[\frac{m}{2}\right]-2-2 l}(x, \xi, \tau)$ is the $2\left[\frac{m}{2}\right]-2 l-2$ order symbol of $P\left(\partial_{t}+F\right)^{-1}$ and the second equality in (2.37) holds in local coordinates.

2.2. The local family index formula. In [Bi2],[BV1],[Do1],[Zh1], several different proofs of the local index theorem for families of Dirac operators were given. In this section, we shall give a new proof of the local family index formula by using the Greiner's approach of the heat kernel asymptotics with form coefficients. Comparing with previous proofs, we use the Volterra calculus with form coefficients instead of heat equation discussions to get the family heat kernel asymototics as in [Po1]. 
Let us introduce some notations. For $z \in B$, denote by $D_{z}$ the restriction of $\mathrm{D}$ to the fiber acting on $\Gamma\left(M_{z}, S\left(T M_{z}\right)\right)$. We set that the dimension $n$ of fibre to be even. Then $\Gamma\left(M_{z}, S\left(T M_{z}\right)\right)$ has a splitting as a sum of $\Gamma^{+}\left(M_{z}, S\left(T M_{z}\right)\right)$ and $\Gamma^{-}\left(M_{z}, S\left(T M_{z}\right)\right)$. The operator $D_{z}$ interchanges $\Gamma^{+}\left(M_{z}, S\left(T M_{z}\right)\right)$ and $\Gamma^{-}\left(M_{z}, S\left(T M_{z}\right)\right)$. Let $D_{z,+}, D_{z,-}$ be the restrictions of $D_{z}$ to $\Gamma^{+}\left(M_{z}, S\left(T M_{z}\right)\right)$ and $\Gamma^{-}\left(M_{z}, S\left(T M_{z}\right)\right)$ respectively. By Chapter 9 in [BGV], the difference bundle $\left[\operatorname{ker} D_{z,+}\right]-\left[\operatorname{ker} D_{z,-}\right]$ over $B$ is well defined in the sense of $K$ theory. The family index theorem presents a calculation of the Chern character of the difference bundle as a differential form over $B$ explicitly. We change the normalization constant in the definition of the Chern character. Namely, for a vector bundle $V$ with connection form $\gamma$ and curvature $C$, we set $\operatorname{Ch}(V)=\operatorname{Tr}(\exp (-C))$. Let $\widehat{A}\left(R^{T Z}\right)=\operatorname{det}^{\frac{1}{2}}\left(\frac{R^{T Z} / 2}{\sinh \left(R^{T Z} / 2\right)}\right)$ and $\int_{M_{z}}$ denote the integral along the fibre. Then we have

Theorem 2.12 (Atiyah-Singer $[\mathrm{AS}]$ ). It holds that the form

$$
(2 i \pi)^{-\frac{n}{2}} \int_{M_{z}} \widehat{A}\left(R^{T Z}\right)
$$

is a representative of $\mathrm{Ch}\left(\left[\operatorname{ker} D_{z,+}\right]-\left[\operatorname{ker} D_{z,-}\right]\right)$.

Let $\bar{F}$ be a complex vector bundle on $M$ and $D_{z}^{\bar{F}}$ be the twisted Dirac operator along the fibre. Denote by $\operatorname{ch}(\bar{F})$ the Chern character of $\bar{F}$, we can get a twisted index bundle and the twisted family index theorem:

Theorem 2.13 (Atiyah-Singer [AS]). It holds that the form

$$
(2 i \pi)^{-\frac{n}{2}} \int_{M_{z}} \widehat{A}\left(R^{T Z}\right) \operatorname{ch}(\bar{F})
$$

is a representative of $\mathrm{Ch}\left(\left[\operatorname{ker} D_{z,+}^{\bar{F}}\right]-\left[\operatorname{ker} D_{z,-}^{\bar{F}}\right]\right)$.

We shall prove Theorem 2.12 and Theorem 2.13 similarly as follows. For $t>0$, we set that $S_{x}$ is the spinor space on $T_{x}\left(M_{z}\right)$ and

$$
\psi_{t}: \operatorname{Hom}\left(S_{x}, S_{x}\right) \widehat{\otimes} \wedge\left(T_{z}^{*} B\right) \rightarrow \operatorname{Hom}\left(S_{x}, S_{x}\right) \widehat{\otimes} \wedge\left(T_{z}^{*} B\right) \quad h d y_{\alpha} \mapsto \frac{1}{\sqrt{t}} h d y_{\alpha} .
$$

For $\omega \in \wedge\left(T_{z}^{*} B\right)$ and $A \in \operatorname{Hom}\left(S_{x}, S_{x}\right)$, we define $\operatorname{Tr}_{\mathrm{s}}(\omega A)=\omega \operatorname{Tr}_{\mathrm{s}}(A)$. By Proposition 4.1 in $[\mathrm{Z} 1]$, we get

Proposition 2.14 ([Bi2, Z1]). For $t>0$,

$$
\psi_{t} \int_{M_{z}} \operatorname{Tr}_{\mathrm{s}} k_{t}^{z}(x, x) d x
$$

is a representative of $\mathrm{Ch}\left(\left[\operatorname{ker} D_{z,+}\right]-\left[\operatorname{ker} D_{z,-}\right]\right)$.

By Proposition 2.14, in order to prove Theorem 2.12, we only need to prove the following local family index formula:

TheOREM 2.15([AS]). We have

$$
\lim _{t \rightarrow 0} \psi_{t} \int_{M_{z}} \operatorname{Tr}_{s} k_{t}^{z}(x, x) d x=(2 i \pi)^{-\frac{n}{2}} \int_{M_{z}} \widehat{A}\left(R^{T Z}\right) .
$$


In order to prove Theorem 2.15, let us recall the symbol map and the Getzler order, then we compute the symbol map of the heat kernel. Let $n$ be even and $\operatorname{End}\left(S\left(T M_{z}\right)\right)$ is a bundle of algebras over $M_{z}$ isomorphic to the Clifford bundle $\mathrm{Cl}\left(M_{z}\right)$, whose fiber $\mathrm{Cl}_{x}\left(M_{z}\right)$ at $x \in M_{z}$ is the complex algebra generated by 1 and elements of $T_{x}^{*} M_{z}$ with relations

$$
\xi \cdot \eta+\eta \cdot \xi=-2\langle\xi, \eta\rangle, \quad \xi, \eta \in T_{x}^{*} M_{z} .
$$

Recall that the quantization map $c: \wedge T_{\mathbb{C}}^{*}\left(M_{z}\right) \rightarrow \mathrm{Cl}(M)$ and the symbol map $\sigma=c^{-1}$ satisfy

$$
\sigma(c(\xi) c(\eta))=\xi \wedge \eta-\langle\xi, \eta\rangle .
$$

So, for $\xi$ and $\eta$ in $\wedge T_{\mathbb{C}}^{*}\left(M_{z}\right)$ we have

$$
\sigma\left(c\left(\xi^{(i)}\right) c\left(\eta^{(j)}\right)\right)=\xi^{(i)} \wedge \eta^{(j)} \bmod \wedge^{i+j-2} T_{\mathbb{C}}^{*}\left(M_{z}\right),
$$

where $\xi^{(l)}$ denotes the component in $\wedge^{l} T_{\mathbb{C}}^{*}\left(M_{z}\right)$ of $\xi \in \wedge T_{\mathbb{C}}^{*}\left(M_{z}\right)$. Since we compute the local index in a fixed fibre $M_{z}$, so we use an observation due to Getzler as in the case of a single manifold. If $e_{1}, \cdots, e_{n}$ is an orthonormal frame of $T_{x} M_{z}$, then

$$
\operatorname{Tr}_{s}\left[c\left(e^{i_{1}}\right) \cdots c\left(e^{i_{k}}\right)\right]= \begin{cases}0 & \text { if } k \neq n \\ (-2 i)^{\frac{n}{2}} & \text { if } k=n .\end{cases}
$$

We shall prove (2.41) at a fixed point $x_{0} \in M_{z}$. Using normal coordinates centered at $x_{0}$ in $M_{z}$ and paralleling $\partial_{i}$ at $x_{0}$ along geodesics through $x_{0}$, we get the orthonormal frame $e_{1}, \cdots, e_{n}$. By

$$
k_{t}^{z}(0,0)=K_{Q}^{z}(0,0, t)+O\left(t^{\infty}\right) \quad \text { as } t \rightarrow 0^{+},
$$

and by (2.45), we get

$$
\lim _{t \rightarrow 0} \psi_{t} \int_{M_{z}} \operatorname{Tr}_{s} k_{t}^{z}(x, x) d x=(-2 i)^{\frac{n}{2}} \lim _{t \rightarrow 0} \int_{M_{z}} \sigma\left[\psi_{t} K_{Q}(0,0, t)\right] .
$$

We define the Getzler order in [Po1] as follows:

$$
\operatorname{deg} \partial_{j}=\frac{1}{2} \operatorname{deg} \partial_{t}=\operatorname{deg} c\left(d x_{j}\right)=\operatorname{deg} c\left(d y_{j}\right)=-\operatorname{deg} x^{j}=1 .
$$

Let $Q \in \Psi_{V}^{*}\left(\mathbb{R}^{n} \times \mathbb{R}, S\left(T M_{z}\right) \otimes \wedge^{*} T_{z}^{*} B\right)$ have symbol

$$
q(x, \xi, \tau) \sim \sum_{k \leq m^{\prime}} \sum_{l=1}^{2^{\bar{q}}} q_{k, l}(x, \xi, \tau) \omega^{[l]},
$$

where $\omega^{[l]} \in \wedge^{l} T_{z}^{*} B$ and $q_{k, l}(x, \xi, \tau)$ is an order $k$ symbol. Then taking components in each subspace $\wedge^{j} T^{*} M_{z}$ and using Taylor expansions at $x=0$ give formal expansions

$$
\sigma[q(x, \xi, \tau)] \sim \sum_{j, k, l} \sigma\left[q_{k, l}(x, \xi, \tau)\right]^{(j)} \omega^{[l]} \sim \sum_{j, k, l, \alpha} \frac{x^{\alpha}}{\alpha !} \sigma\left[\partial_{x}^{\alpha} q_{k, l}(0, \xi, \tau)\right]^{(j)} \omega^{[l]} .
$$

The symbol $\frac{x^{\alpha}}{\alpha !} \sigma\left[\partial_{x}^{\alpha} q_{k, l}(0, \xi, \tau)\right]^{(j)} \omega^{[l]}$ is Getzler homogeneous of order $k+j+l-|\alpha|$. So we can expand $\sigma[q(x, \xi, \tau)]$ as

$$
\sigma[q(x, \xi, \tau)] \sim \sum_{j \geq 0} q_{(m-j)}(x, \xi, \tau), \quad q_{(m)} \neq 0,
$$


where $q_{(m-j)}$ is a Getzler homogeneous symbol of degree $m-j$.

Definition 2.16 ([Po1]). The integer $m$ is called the Getzler order of $Q$. The symbol $q_{(m)}$ is the principle Getzler homogeneous symbol of $Q$. The operator $Q_{(m)}=$ $q_{(m)}\left(x, D_{x}, D_{t}\right)$ is called the model operator of $Q$.

Denote by $O\left(t^{\frac{k}{2}}\right)$ a Laurant expansion about $t^{\frac{1}{2}}$ whose lowest degree about $t$ is $\frac{k}{2}$.

Lemma 2.17. Let $Q \in \Psi_{V}^{*}\left(\mathbb{R}^{n} \times \mathbb{R}, S\left(T\left(M_{z}\right)\right) \otimes \wedge^{*} T_{z}^{*} B\right)$ have Getzler order $m$ and model operator $Q_{(m)}$. Then as $t \rightarrow 0^{+}$we have:

$$
\begin{gathered}
\sigma\left[\psi_{t} K_{Q}(0,0, t)\right]^{(j)}=\omega^{\text {odd }} O\left(t^{\frac{j-n-m-2}{2}}\right)+O\left(t^{\frac{j-n-m-1}{2}}\right), \quad \text { if } m-j \text { is odd } \\
\sigma\left[\psi_{t} K_{Q}(0,0, t)\right]^{(j)}=t^{\frac{j-n-m-2}{2}} K_{Q_{(m)}}(0,0,1)^{(j)}+\omega_{1}^{\text {odd }} O\left(t^{\frac{j-n-m-1}{2}}\right)+O\left(t^{\frac{j-n-m}{2}}\right),
\end{gathered}
$$

if $m-j$ is even, where $\sigma\left[K_{Q}(0,0, t)\right]^{(j)}$ denotes the degree $j$ form component in $M_{z}$ and $\omega^{\text {odd }}, \omega_{1}^{\text {odd }}$ are in $\wedge^{\text {odd }}\left(T^{*} B\right) \otimes \wedge\left(T^{*}\left(M_{z}\right)\right)$. In particular $m=-2$ and $j=n$ is even, we get

$$
\sigma\left[\psi_{t} K_{Q}(0,0, t)\right]^{(n)}=K_{Q_{(-2)}}(0,0,1)^{(n)}+O(t)
$$

Proof. By (1.7) in [Po1], we get

$$
K_{Q}(0,0, t) \sim \sum_{m_{0}-j_{0} \text { even }} \sum_{l} t^{\frac{j_{0}-n-m_{0}-2}{2}} \check{q}_{m_{0}-j_{0}, l}(0,0,1) \omega^{[l]},
$$

where $m_{0}$ is the operator order of $Q$. And then

$$
\sigma\left[\psi_{t} K_{Q}(0,0, t)\right]^{(j)} \sim \sum_{m_{0}-j_{0} \text { even }} \sum_{l} t^{\frac{j_{0}-n-m_{0}-l-2}{2}} \sigma\left[\check{q}_{m_{0}-j_{0}, l}(0,0,1)\right]^{(j)} \omega^{[l]} .
$$

Let $L=m_{0}-j_{0}+j+l$. By $Q$ having the Getzler order $m$, then $L \leq m$. Then

$$
\sigma\left[\psi_{t} K_{Q}(0,0, t)\right]^{(j)} \sim \sum_{m_{0}-j_{0} \text { even }} \sum_{l} \sum_{L \leq m} t^{\frac{j-n-L-2}{2}} \sigma\left[\check{q}_{m_{0}-j_{0}, l}(0,0,1)\right]^{(j)} \omega^{[l]} .
$$

We note that the leading term degree is $L=m$ and $m_{0}-j_{0}+l=m-j$. When $m-j$ is odd, since $m_{0}-j_{0}$ is even, then $l$ is odd, then we get

$$
\sigma\left[\psi_{t} K_{Q}(0,0, t)\right]^{(j)}=\omega^{\text {odd }} O\left(t^{\frac{j-n-m-2}{2}}\right)+O\left(t^{\frac{j-n-m-1}{2}}\right) .
$$

When $L=m$ and $m-j$ are even, $l$ is even. In this case, the leading coefficient is

$$
\sigma\left[\breve{q}_{(m)}(0,0,1)\right]^{(j)}=\sum_{l} \sigma\left[\breve{q}_{m-j-l, l}(0,0,1)\right]^{(j)} \omega^{[l]}=K_{Q_{(m)}}(0,0,1)^{(j)} .
$$

In the next term, $L=m-1$ and $m-j$ are even and $m_{0}-j_{0}+l+j=m-1$, then $l$ is odd, so $m-j-l$ is odd and $\breve{q}_{m-j-l, l}(0,0,1)=0$. Then the next term is $O\left(t^{\frac{j-n-m}{2}}\right)$.

In the sequel we say that a symbol or a $\Psi D O$ is $O_{G}(m)$ if it has Getzler order $\leq m$. Similar to Lemma 4 in [Po1], we have: 
Lemma 2.18. For $j=1,2$, let $Q_{j} \in \Psi_{V}^{*}\left(\mathbb{R}^{n} \times \mathbb{R}, S\left(T\left(M_{z}\right)\right) \otimes \wedge^{*} T_{z}^{*} B\right)$ have Getzler order $m_{j}$ and model operator $Q_{\left(m_{j}\right)}$ and assume that either $Q_{1}$ or $Q_{2}$ is properly supported. Then we have:

$$
Q_{1} Q_{2}=c\left[Q_{\left(m_{1}\right)} Q_{\left(m_{2}\right)}\right]+O_{G}\left(m_{1}+m_{2}-1\right) .
$$

Proof. Let $Q_{j}=\omega_{j} \widehat{Q}_{j}$ for $j=1,2$ where $\omega_{j} \in \wedge^{l_{j}}\left(T_{z}^{*} B\right)$ and $\widehat{Q}_{j} \in \Psi_{V}^{*}\left(\mathbb{R}^{n} \times\right.$ $\mathbb{R}, S\left(T\left(M_{z}\right)\right)$ has the Getzler order $m_{j}-l_{j}$. Then

$$
Q_{1} Q_{2}=\omega_{1} \wedge \omega_{2} \widehat{Q}_{1} \widehat{Q}_{2} .
$$

By Lemma 4 in [Po1], we have

$$
\widehat{Q}_{1} \widehat{Q}_{2}=c\left[\widehat{Q}_{\left(m_{1}-l_{1}\right)} \widehat{Q}_{\left(m_{2}-l_{2}\right)}\right]+O_{G}\left(m_{1}+m_{2}-l_{1}-l_{2}-1\right) .
$$

By (2.58) and (2.59), we get (2.57).

In the following, we compute the model operator of $F$ in (2.5). Let $x_{0}, x \in M_{z}$ and $\tau^{E}\left(x_{0}, x\right)$ be the parallel transport map in the bundle $\pi^{*} \wedge^{*}\left(T_{z}^{*} B\right) \otimes S\left(T M_{z}\right)$ along the geodesic from $x$ to $x_{0}$, defined with respect to the Clifford connection $\nabla^{E, 0}$. Using this map, we can trivialize the bundle $\pi^{*} \wedge^{*}\left(T_{z}^{*} B\right) \otimes S\left(T M_{z}\right)$. We note that $e_{1}, \cdots, e_{n}$ is the parallel transport frame with respect to $\nabla^{T Z}$ and so $m_{0}\left(e_{i}\right)$ is not a constant matrix under above trivialization. But we have

Leмma 2.19. ([BGV Lemma 10.25]) If $c^{i}$ is the Clifford action of the cotangent vector $d x_{i}$ acting on $E$ at $x_{0}$ and $\varepsilon^{\alpha}$ is multiplication by $f^{\alpha}$ in the exterior algebra $\wedge^{*}\left(T_{z}^{*} B\right)$, we have

$$
m_{0}\left(e^{i}\right)=c^{i}+\sum u_{\alpha}^{i} \varepsilon^{\alpha} ; \quad m_{0}\left(f^{\alpha}\right)=\varepsilon^{\alpha},
$$

where $u_{\alpha}^{i}$ are smooth functions on $U$ satisfying $u_{\alpha}^{i}(x)=O(|x|)$.

LEMMA 2.20 ([BGV Lemma 10.26]). In the trivialization of $E$ over $U$ induced by the parallel transport map $\tau^{E}\left(x_{0}, x\right)$, the connection $\nabla^{E, 0}$ equals $d+\Theta$, where

$$
\Theta\left(\partial_{i}\right)=-\frac{1}{4} \sum_{j, a<b}\left\langle R\left(\partial_{i}, \partial_{j}\right) e_{a}, e_{b}\right\rangle m^{a} m^{b} x^{j}+\sum_{a<b} f_{i a b}(x) m^{a} m^{b}+g_{i}(x) ;
$$

here $m^{a}$ represents $c^{i}$ or $\varepsilon^{\alpha}$ and $f_{i a b}=O\left(|x|^{2}\right)$ and $g_{i}(x)=O(|x|)$.

By (2.5) and Lemma 2.19 and Lemma 2.20, we get

Proposition 2.21. In the trivialization of $E$ over $U$ induced by the parallel transport map $\tau^{E}\left(x_{0}, x\right)$ and the normal coordinates, the model operator of $F$ is

$$
F_{(2)}=-\sum_{i=1}^{n}\left(\partial_{i}-\frac{1}{4} \sum_{j=1}^{n} a_{i j} x_{j}\right)^{2}, \quad a_{i j}=\left\langle R^{T Z} \partial_{i}, \partial_{j}\right\rangle .
$$

We note that $\lim _{t \rightarrow 0} \psi_{t} \int_{M_{z}} \operatorname{Tr}_{s} k_{t}^{z}(x, x) d x$ does not depend on the trivialization. Using the same discussions with Lemma 5 and Lemma 6 in [Po1], we get

Lemma 2.22. Let $Q$ be a Volterra paramatrix for $F+\partial_{t}$. Then $Q$ has the model operator $\left(F_{(2)}+\partial_{t}\right)^{-1}$ and

$$
K_{\left(F_{(2)}+\partial_{t}\right)^{-1}}(0,0,1)=(4 \pi)^{-\frac{n}{2}} \widehat{A}\left(R^{T Z}\right) .
$$

By (2.46), (2.51) and Lemma 2.22, we proved Theorem 2.15. 
3. The local equivariant family index theorem. In $[\mathrm{LM}]$, Liu and Ma gave a proof of the local equivariant family index theorem by the finite propagation speed method in [B3]. In [PW], a new proof of the local equivariant index theorem was given by the Volterra calculus. In this section, we shall give a new proof of the local equivariant family index theorem by the Volterra calculus.

Let us give the fundamental setup. We assume that $M$ and $T Z$ are oriented and $G$ is a compact Lie group which is a fiberwise isometry on $M$ and preserves the orientation of $T Z$. Then $G$ acts as identity on $B$. We also assume that the action of $\mathrm{G}$ lifts to $S(T Z)$ and that the $G$-action commutes with $D$. By Proposition 1.1 in [LM], we know that $\operatorname{Ind} D^{G} \in K_{G}(B)$. Now let us calculate the equivariant Chern character $\operatorname{ch}_{\phi}\left(\operatorname{Ind}\left(\mathrm{D}^{\mathrm{G}}\right)\right)$ in terms of the fixed point data of $\phi \in G$. Set $M^{\phi}=\{x \in M, \phi x=x\}$. Then $\pi: M^{\phi} \rightarrow B$ is a fibration with compact fibre $M_{z}^{\phi}$. By [BGV, Proposition 6.14], $T Z^{\phi}$ is naturally oriented in $M^{\phi}$. Let $N$ denote the normal bundle of $M^{\phi}$, then $N=$ $T Z / T Z^{\phi}$. We fixed a fibre $M_{z}$ and denote by $\widetilde{\phi}$ the lift of $\phi$ which maps $S\left(T\left(M_{z}\right)_{x}\right)$ to $S\left(T\left(M_{z}\right)_{\phi x}\right)$. We denote by $M_{z}^{\phi}$ the fixed-point set of $\phi$, and for $a=0, \cdots, n$, we let $M_{z}^{\phi}=\bigcup_{0 \leq a \leq n} M_{z, a}^{\phi}$, where $M_{z, a}^{\phi}$ is an $a$-dimensional submanifold. Given a fixed-point $x_{0}$ in a component $M_{z, a}^{\phi}$, consider some local coordinates $x=\left(x^{1}, \cdots, x^{a}\right)$ around $x_{0}$. Setting $b=n-a$, we may further assume that over the range of the domain of the local coordinates there is an orthonormal frame $e_{1}(x), \cdots, e_{b}(x)$ of $N_{z}^{\phi}$. This defines fiber coordinates $v=\left(v_{1}, \cdots, v_{b}\right)$. Composing with the map $(x, v) \in N_{z}^{\phi}\left(\varepsilon_{0}\right) \rightarrow \exp _{x}(v)$ we then get local coordinates $x^{1}, \cdots, x^{a}, v^{1}, \cdots, v^{b}$ for $M_{z}$ near the fixed point $x_{0}$. We shall refer to this type of coordinates as tubular coordinates. Then $N_{z}^{\phi}\left(\varepsilon_{0}\right)$ is homeomorphic with a tubular neighborhood of $M_{z}^{\phi}$. We have

TheOrem 3.1 ([LM]). For any $t>0$, the form $\operatorname{Str}\left[\widetilde{\phi} \psi_{t} \exp (-t F)\right]$ is closed and its de-Rham cohomology class in $B$ is independent of $t$ and represents $\operatorname{ch}_{\phi}\left(\operatorname{Ind}\left(D^{G}\right)\right)$ in the de-Rham cohomology of $B$.

We shall use Theorem 3.1 to find a local index formula for $\operatorname{ch}_{\phi}\left(\operatorname{Ind}\left(D^{G}\right)\right)$ by estimating $\operatorname{Str}\left[\widetilde{\phi} \psi_{t} \exp (-t F)\right]$. By the Mckean-Singer formula, we have

$$
\begin{aligned}
\operatorname{Str}\left[\widetilde{\phi} \psi_{t} \exp (-t F)\right] & =\psi_{t} \int_{M_{z}} \operatorname{Str}\left[\widetilde{\phi} k_{t}^{z}(x, \phi(x))\right] d x \\
& =\psi_{t} \int_{M_{z}} \operatorname{Str}\left[\widetilde{\phi} K_{\left(F+\partial_{t}\right)^{-1}}^{z}(x, \phi(x), t)\right] d x .
\end{aligned}
$$

Let $Q=\left(F+\partial_{t}\right)^{-2}$. For $x \in M_{z}^{\phi}$ and $t>0$ set

$$
I_{Q}(x, t):=\widetilde{\phi}(x)^{-1} \int_{N_{x}^{\phi}(\varepsilon)} \widetilde{\phi}\left(\exp _{x} v\right) K_{Q}\left(\exp _{x} v, \exp _{x}\left(\phi^{\prime}(x) v\right), t\right) d v .
$$

Here we use the trivialization of $S\left(T M_{z}\right)$ about the tubular coordinates. Using the tubular coordinates, then

$$
I_{Q}(x, t)=\int_{|v|<\varepsilon} \widetilde{\phi}(x, 0)^{-1} \widetilde{\phi}(x, v) K_{Q}\left(x, v ; x, \phi^{\prime}(x) v ; t\right) d v .
$$

Let

$$
q_{m-j}^{E}(x, v ; \xi, \nu ; \tau):=\widetilde{\phi}(x, 0)^{-1} \widetilde{\phi}(x, v) q_{m-j}(x, v ; \xi, \nu ; \tau) .
$$

Using the same proof as Lemma 2.9, we obtain the following proposition by Lemma 8.2 and Lemma 8.3 in $[\mathrm{PW}]$ 
Proposition 3.2. Let $Q \in \Psi_{V}^{m}\left(M_{z} \times \mathbb{R}, E\right), m \in \mathbb{Z}$. Uniformly on each component $M_{z, a}^{\phi}$

$$
I_{Q}(x, t) \sim \sum_{j \geq 0} t^{-\left(\frac{a}{2}+\left[\frac{m}{2}\right]+1\right)} I_{Q}^{j}(x) \quad \text { as } t \rightarrow 0^{+},
$$

where $I_{Q}^{j}(x)$ is defined in tubular coordinates by

$$
I_{Q}^{(j)}(x):=\sum_{|\alpha| \leq m-\left[\frac{m}{2}\right]+2 j} \int \frac{v^{\alpha}}{\alpha !}\left(\partial_{v}^{\alpha} q_{2\left[\frac{m}{2}\right]-2 j+|\alpha|}^{E}\right)^{\vee}\left(x, 0 ; 0,\left(1-\phi^{\prime}(x)\right) v ; 1\right) d v .
$$

By Proposition 8.7 in [PW] and Proposition 3.2 and the definition of $\psi_{t}$, we have

Proposition 3.3. Let $P: C^{\infty}\left(M_{z}, \pi^{*} \wedge T_{z}^{*} B \otimes S\left(T\left(M_{z}\right)\right)\right) \rightarrow C^{\infty}\left(M_{z}, \pi^{*} \wedge\right.$ $\left.T_{z}^{*} B \otimes S\left(T\left(M_{z}\right)\right)\right)$ be a differential operator of order $m$.

(1) Uniformly on each component $M_{z, a}^{\phi}$,

$$
I_{P\left(F+\partial_{t}\right)^{-1}}(x, t) \sim \sum_{j \geq 0} t^{-\left(\frac{n}{2}+\left[\frac{m}{2}\right]\right)+j} I_{P\left(F+\partial_{t}\right)^{-1}}^{(j)}(x) \quad \text { as } t \rightarrow 0^{+},
$$

(2) As $t \rightarrow 0^{+}$, we have

$$
\psi_{t} \operatorname{Str}\left[\widetilde{\phi} P e^{-t F}\right] \sim \sum_{0 \leq a \leq n} \sum_{j \geq 0} \sum_{l=1}^{2^{\bar{q}}} t^{-\left(\frac{a}{2}+\left[\frac{m}{2}\right]+\frac{l}{2}\right)+j} \int_{M_{z, a}^{\phi}} \operatorname{Str}\left[\widetilde{\phi} I_{P\left(F+\partial_{t}\right)^{-1}}^{(j), l}(x)\right] d x,
$$

where $I_{P\left(F+\partial_{t}\right)^{-1}}^{(j), l}(x)$ denotes the l-degree component of $I_{P\left(F+\partial_{t}\right)^{-1}}^{(j)}(x)$ in $\wedge T_{z}^{*} B$.

Let

$$
\widehat{A}\left(R^{T Z^{\phi}}\right)=\operatorname{det}^{\frac{1}{2}}\left(\frac{R^{T Z^{\phi}} / 2}{\sinh \left(R^{T Z^{\phi}} / 2\right)}\right) ; \quad \nu_{\phi}\left(R^{N^{\phi}}\right):=\operatorname{det}^{-\frac{1}{2}}\left(1-\phi^{N} e^{-R^{N^{\phi}}}\right) .
$$

For a top degree form $\omega \in C^{\infty}\left(M_{z, a}, \wedge T_{z}^{*}(B) \otimes \wedge^{a} T^{*} M_{z, a}\right)$ in $M_{z, a}$ with coefficients in $\wedge T_{z}^{*}(B)$, we denote by $|\omega|^{(a)}$ the Berezin integral which in $\wedge T_{z}^{*}(B)$, i.e. its inner-product with the volume form of $M_{z, a}$.

Theorem 3.4 (Local equivariant family index theorem). Let $x_{0} \in M^{\phi}$, then

$$
\lim _{t \rightarrow 0} \operatorname{Str}\left[\widetilde{\phi}\left(x_{0}\right) \psi_{t} I_{\left(F+\partial_{t}\right)^{-1}}\left(x_{0}, t\right)\right]=(-i)^{\frac{n}{2}}(2 \pi)^{-\frac{a}{2}}\left|\widehat{A}\left(R^{T Z^{\phi}}\right) \nu_{\phi}\left(R^{N^{\phi}}\right)\right|^{(a)}
$$

REMARK. It is easy to generalize Theorem 3.4 to the twisted $\phi$-complex vector bundle case.

By (3.1) and Lemma 8.1 in $[\mathrm{PW}]$, as $t \rightarrow 0^{+}$

$$
\psi_{t} \int_{M_{z}} \operatorname{Str}\left[\widetilde{\phi} K_{\left(F+\partial_{t}\right)^{-1}}(x, \phi(x), t)\right] d x=\psi_{t} \int_{M_{z}} \operatorname{Str}\left[\widetilde{\phi} I_{Q}(x, \phi(x), t)\right] d x,
$$

By Theorem 3.1, Theorem 3.4 and (3.1), (3.11), we get a representative of $\operatorname{ch}_{\phi}\left(\operatorname{Ind} D^{G}\right)$.

In order to prove Theorem 3.4, we shall compute $\widetilde{\phi}$ in tubular coordinates. Let 
$e_{1}, \ldots, e_{n}$ be an oriented orthonormal basis of $T_{x_{0}} M_{z}$ such that $e_{1}, \cdots, e_{a} \operatorname{span} T_{x_{0}} M_{z}^{\phi}$ and $e_{a+1}, \cdots, e_{n} \operatorname{span} N_{x_{0}}^{\phi}$. This provides us with normal coordinates $\left(x_{1}, \cdots, x_{n}\right) \rightarrow$ $\exp _{x_{0}}\left(x^{1} e_{1}+\cdots+x^{n} e_{n}\right)$. Moreover using parallel translation enables us to construct a synchronous local oriented tangent frame $e_{1}(x), \ldots, e_{n}(x)$ such that $e_{1}(x), \cdots, e_{a}(x)$ form an oriented frame of $T M_{z, a}^{\phi}$ and $e_{a+1}(x), \cdots, e_{n}(x)$ form an (oriented) frame $N^{\phi}$ (when both frames are restricted to $M_{z}^{\phi}$ ). This gives rise to trivializations of the tangent and spinor bundles. Write

$$
\phi^{\prime}(0)=\left(\begin{array}{cc}
1 & 0 \\
0 & \phi^{N}
\end{array}\right) .
$$

Let $\wedge(n)=\wedge_{\mathbb{C}}^{*} \mathbb{R}^{n}$ be the complexified exterior algebra of $\mathbb{R}^{n}$.We shall use the following gradings on $\wedge(n)$,

$$
\wedge(n)=\bigoplus_{1 \leq j \leq n} \wedge^{j}(n)=\bigoplus_{\substack{1 \leq k \leq a \\ 1 \leq \bar{l} \leq b}} \wedge^{k, \bar{l}}(n),
$$

where $\wedge^{j}(n)$ is the space of forms of degree $j$ and $\wedge^{k, \bar{l}}(n)$ is the space of forms $d x^{i_{1}} \wedge$ $\cdots \wedge d x^{i_{k+\bar{l}}}$ with $1 \leq i_{1}<\cdots<i_{k} \leq a$ and $a+1 \leq i_{k+1}<\cdots<i_{k+\bar{l}} \leq n$. Given a form $\omega \in \wedge(n)$ we shall denote by $\omega^{(j)}$ (resp., $\left.\omega^{(k, \bar{l})}\right)$ its component in $\wedge^{j}(n)\left(\right.$ resp., $\wedge^{k, \bar{l}}(n)$ ). We denote by $|\omega|^{(a, 0)}$ the Berezin integral $\left|\omega^{(*, 0)}\right|^{(a, 0)}$ of its component $\omega^{(*, 0)}$ in $\wedge^{(*, 0)}(n)$. Then we have

LEMma 3.5 ([PW,Lemma 9.5]). Let $S_{n}$ be the spinor space associated to $\mathbb{R}^{n}$ and $A \in \operatorname{End}\left(S_{n}\right)$, then

$$
\begin{aligned}
\operatorname{Str}[\widetilde{\phi} A]= & (-2 i)^{\frac{n}{2}} 2^{-\frac{b}{2}} \operatorname{det}^{\frac{1}{2}}\left(1-\phi^{N}\right)|\sigma(A)|^{(a, 0)} \\
& +(-2 i)^{\frac{n}{2}} \sum_{0 \leq b^{\prime}<b}\left|\sigma(\widetilde{\phi})^{\left(0, b^{\prime}\right)} \sigma(A)^{\left(a, b-b^{\prime}\right)}\right|^{(n)} .
\end{aligned}
$$

Similar to Proposition 2.21, we get the same expression of the model operator of $F$ in the trivialization of $E$ over $U$ induced by the parallel transport map $\tau\left(x_{0}, x\right)$ about $\nabla^{T Z}$ and the normal coordinate. We will compute the local index in this trivialization.

Lemma 3.6. Let the operator $Q \in \Psi_{V}^{*}\left(\mathbb{R}^{n} \times \mathbb{R}, S\right) \otimes \wedge\left(T_{z}^{*} B\right)$ have the Getzler order $m$ and model operator $Q_{(m)}$. Then as $t \rightarrow 0^{+}$

(1) $\sigma\left[\psi_{t} I_{Q}(0, t)\right]^{(j)}=\omega^{\text {odd }} O\left(t^{\frac{j-m-a-2}{2}}\right)+O\left(t^{\frac{j-m-a-1}{2}}\right)$ if $m-j$ is odd.

(2) $\sigma\left[\psi_{t} I_{Q}(0, t)\right]^{(j)}=O\left(t^{\frac{j-m-a-2}{2}}\right) I_{Q(m)}(0,1)^{(j)}+\omega_{1}^{\text {odd }} O\left(t^{\frac{j-m-a-1}{2}}\right)+O\left(t^{\frac{j-m-a}{2}}\right)$ if $m-j$ is even.

In particular, for $m=-2$ and $j=a$ we get

$$
\sigma\left[\psi_{t} I_{Q}(0, t)\right]^{(a, 0)}=I_{Q(-2)}(0,1)^{(a, 0)}+O\left(t^{\frac{1}{2}}\right) .
$$

Proof. By the change of variable formula for symbol, similar to (9.23) in [PW], we have

$$
\sigma\left[\psi_{t} I_{Q}(0, t)\right]^{(j)} \sim \sum_{\substack{|\alpha|+|\beta|-|\gamma|-\bar{l} \text { even } \\ \overline{1} \leq m^{\prime}, 2|\gamma|<|\beta|}} \sum_{l=1}^{2^{\bar{q}}} t^{\frac{|\alpha|+|\beta|-|\gamma|-l-\bar{l}-(a+2)}{2}} I_{\bar{l} \alpha \beta \gamma, l}^{(j)},
$$


where $m^{\prime}$ is the operator order of $Q$ and

$$
I_{\bar{l} \alpha \beta \gamma, l}^{(j)}=\int_{\mathbb{R}^{b}} a_{\beta \gamma, \bar{l}}(0, v) \frac{v^{\alpha}}{\alpha !}\left(\partial_{v}^{\alpha} \sigma\left[\xi^{\gamma} D_{\xi}^{\beta} q_{\bar{l}, l}\right]^{(j)}\right)^{\vee}\left(0,0 ; 0,\left(1-\phi^{N}(0)\right) v ; 1\right) d v \omega^{[l]},
$$

where $a_{\beta \gamma, \bar{l}}\left(x^{\prime}, v\right)$ are smooth functions such that $a_{\beta \gamma, \bar{l}}(x)=1$ when $\beta=\gamma=0$. Since $Q$ has the Getzler order $m$, then all the coefficients $I_{\bar{l} \alpha \beta \gamma, l}^{(j)}$ with $\bar{l}+j+l-|\alpha|>m$ are zero. So, if $\bar{l}+j+l-|\alpha| \leq m$ and $2|\gamma| \leq|\beta|$, then $t^{\frac{|\alpha|+|\beta|-|\gamma|-l-\bar{l}-(a+2)}{2}}$ is $O\left(t^{\frac{j-m-a-2}{2}}\right)$ and even is $o\left(t^{\frac{j-m-a-2}{2}}\right)$ if we have $\bar{l}+j+l-|\alpha|<m$ or $(\beta, \gamma) \neq(0,0)$.

Observe that terms in asymptotic (3.14) containing integer powers (resp. half integer powers) of $t$ when $l$ is even (resp. odd) since $a$ is even. When $m-j$ is odd, the leading term is $O\left(t^{\frac{j-m-a-2}{2}}\right)$ which is a half integer, so its coefficient is in $\Omega^{\text {odd }}(B)$ and (1) is verified. When $m-j$ is even, similar discussions show that

$$
\sigma\left[\psi_{t} I_{Q}(0, t)\right]^{(j)}=\omega^{\text {even }} O\left(t^{\frac{j-m-a-2}{2}}\right)+\omega^{\text {odd }} O\left(t^{\frac{j-m-a-1}{2}}\right)+O\left(t^{\frac{j-m-a}{2}}\right) .
$$

Then similar to the discussions in $[\mathrm{PW}]$, we prove (2).

By (3.12) and Lemma 3.6 (2) and (3.13) and Lemma 9.13 in [PW], we get Theorem 3.4 .

4. The equivariant JLO character for a family of the Dirac operators. In $[\mathrm{CH}]$, Chern and $\mathrm{Hu}$ computed the equivariant JLO characters for invariant Dirac operators. In [Az2], Azmi computed the JLO characters for a family of Dirac operators. In [Az3], Azmi constructed an equivariant bivariant cyclic theory, as a combination of equivariant cyclic and noncommutative de Rham theories for unital $G$-Banach algebras, where $G$ is a compact Lie group. By incorporating the JLO formula and the superconnection formalism of Quillen, an equivariant bivariant JLO character of Kasparov's G-bimodule is defined, with values in the bivariant cyclic theory. In this section, following the ideas in $[\mathrm{CH}],[\mathrm{Zh} 2]$ and $[\mathrm{Fe}]$, we compute the equivariant JLO characters for a family of the Dirac operators.

Let $C^{1}(M)$ be the Banach algebra which is the completion of $C^{\infty}(M)$ with respect to the norm $|f|:=\|f\|+\|[D, f]\|$, for $f \in C^{\infty}(M)$. The commutator $[D, f]$ extends to a bounded operator on $\mathcal{H}=L^{2}(M, S(T Z))$. The algebra $C^{1}(M)$ acts on $L(\mathcal{H})$

(bounded operators on $\mathcal{H}$ ) by multiplication. Denote by $\Delta$ the projective tensor product of the Banach algebras $C^{1}(M)$ and $C^{\infty}(B)$, i.e $\Delta=C^{\infty}(B) \hat{\otimes} C^{1}(M)$, with the projective tensor product norm.

Let $\mathcal{M}=\wedge B \otimes \mathcal{H}$ be a $\Delta-C^{\infty}(B)$ bimodule, where $\Delta$ acts on the left of $\mathcal{M}$ by letting $C^{\infty}(B)$ act on $\wedge(B)$ by multiplication by left and $C^{1}(M)$ acts on $\mathcal{H}$ while $C^{\infty}(B)$ acts on $\mathcal{M}$ by multiplication from right. There is a continuous $C^{\infty}(B)$-valued inner product on $\mathcal{M}$. There is an obvious continuous action of $\phi \in G$ on $\mathcal{M}$, by letting $\phi$ act on $\mathcal{H}$ via $\widetilde{\phi}$ and on $\wedge B$ via the identity map. Let $\mathcal{B}_{t}=\sqrt{t} \psi_{t}(\mathcal{B})$ be the rescaled Bismut' superconnection with the rescaled curvature $F_{t}=\mathcal{B}_{t}^{2}=t \psi_{t}(F)$. If $h_{i} \otimes f_{i} \in \Delta, 0 \leq i \leq 2 k$ are operators on $\mathcal{M}$, we define the equivariant bivariant JLO character by:

$$
\begin{aligned}
& \mathrm{Ch}_{2 k}\left(\mathcal{B}_{t}\right)(\phi)\left(h_{0} \otimes f_{0}, \cdots, h_{2 k} \otimes f_{2 k}\right) \\
= & t^{k} \int_{\triangle_{2 k}} \operatorname{Str}\left[\psi_{t} \widetilde{\phi} h_{0} \otimes f_{0} e^{-t s_{1} F}\right. \\
& \left.\cdot\left[\mathcal{B}, h_{1} \otimes f_{1}\right] e^{-t\left(s_{2}-s_{1}\right) F} \cdots\left[\mathcal{B}, h_{2 k} \otimes f_{2 k}\right] e^{-t\left(1-s_{2 k}\right) F}\right] d s,
\end{aligned}
$$


where $\triangle_{2 k}=\left\{\left(s_{1}, \cdots, s_{2 k}\right) \mid 0 \leq s_{1} \leq \cdots \leq s_{2 k} \leq 1\right\}$ is the simplex in $\mathbb{R}^{2 k}$. We will compute

$$
\lim _{t \rightarrow 0} \mathrm{Ch}_{2 k}\left(\mathcal{B}_{t}\right)(\phi) .
$$

In the following, we give some estimates about $\mathrm{Ch}_{2 k}\left(\mathcal{B}_{t}\right)(\phi)$. Since $B$ is compact, we can fix a fibre $M_{z}$ and estimate $\mathrm{Ch}_{2 k}\left(\mathcal{B}_{t}\right)(\phi)$. Let $H$ be a Hilbert space. For $q \geq 0$, denote by $\|.\|_{q}$ the Schatten $p$-norm on the Schatten ideal $L^{p}$. Denote by $L(H)$ the Banach algebra of bounded operators on $H$.

Lemma 4.1 ([Si]). (i) $\operatorname{Tr}(A B)=\operatorname{Tr}(B A)$, for $A, B \in L(H)$ and $A B, B A \in L^{1}$. (ii) For $A \in L^{1}$, we have $|\operatorname{Tr}(A)| \leq\|A\|_{1},\|A\| \leq\|A\|_{1}$.

(iii) For $A \in L^{q}$ and $B \in L(H)$, we have: $\|A B\|_{q} \leq\|B\|\|A\|_{q},\|B A\|_{q} \leq\|B\|\|\mid\|_{q_{q}}$. (iv) (Hölder Inequality) If $\frac{1}{r}=\frac{1}{p}+\frac{1}{q}, p, q, r>0, A \in L^{p}, B \in L^{q}$, then $A B \in L^{r}$ and $\|A B\|_{r} \leq\|A\|_{p}\|B\|_{q}$.

Lemma 4.2. For any $u>0, t>0$ and $t$ is small and any order $l$ fibrewise differential operator $\bar{B}$ with form coefficients, we have:

$$
\left\|e^{-u t F} \bar{B}\right\|_{u^{-1}} \leq C_{l} u^{-\frac{l}{2}} t^{-\frac{l}{2}}\left(\operatorname{tr}\left[e^{-\frac{t D^{2}}{2}}\right]\right)^{u} .
$$

Proof. By (2.7), we have

$$
\begin{aligned}
\left\|e^{-u t F} \bar{B}\right\|_{u^{-1}}= & \| \sum_{m \geq 0}(-u t)^{m} \int_{\triangle_{m}} e^{-v_{0} u t D^{2}} F_{[+]} e^{-v_{1} u t D^{2}} \\
& \cdot F_{[+]} \cdots e^{-v_{m-1} u t D^{2}} F_{[+]} e^{-v_{m} u t D^{2}} \bar{B} d v \|_{u^{-1}},
\end{aligned}
$$

We estimate the term of $m=2$ in the right hand of (4.3), other terms are similar. We split $\triangle(2)=J_{0} \cup J_{1} \cup J_{2}$ where $J_{i}=\left\{\left(v_{0}, v_{1}, v_{2}\right) \in \Delta(2) \mid v_{i} \geq \frac{1}{3}\right\}$.

$$
\begin{aligned}
& (u t)^{2}\left\|\int_{J_{0}} e^{-v_{0} u t D^{2}} F_{[+]} e^{-v_{1} u t D^{2}} F_{[+]} e^{-v_{2} u t D^{2}} \bar{B} d v\right\|_{u^{-1}} \\
\leq & (u t)^{2} \int_{J_{0}}\left\|e^{-\frac{v_{0} u t}{2} D^{2}}\right\|_{\left(u v_{0}\right)^{-1}}\left\|e^{-\frac{v_{0} u t}{2} D^{2}}\left(1+D^{2}\right)^{\frac{l+2}{2}}\right\|\left\|\left(1+D^{2}\right)^{-\frac{l+2}{2}} F_{[+]}\left(1+D^{2}\right)^{\frac{l+1}{2}}\right\| \\
& \left.\cdot\left\|e^{-v_{1} u t D^{2}}\right\|\left\|_{\left(u v_{1}\right)^{-1}}\right\|\left(1+D^{2}\right)^{-\frac{l+1}{2}} F_{[+]}\left(1+D^{2}\right)^{\frac{l}{2}}\|\| e^{-v_{2} u t D^{2}}\left\|_{\left(u v_{2}\right)-1}\right\| 1+D^{2}\right)^{-\frac{l}{2}} \bar{B} \| d v \\
\leq & (u t)^{2} \int_{J_{0}}\left(\operatorname{Tr} e^{-\frac{t}{2} D^{2}}\right)^{u v_{0}}\left(\operatorname{Tr} e^{-t D^{2}}\right)^{u\left(v_{1}+v_{2}\right)}\left(u v_{0} t\right)^{-\frac{l+2}{2}} \\
& \left.\cdot\left\|\left(1+D^{2}\right)^{-\frac{l+2}{2}} F_{[+]}\left(1+D^{2}\right)^{\frac{l+1}{2}}\right\|\left\|\left(1+D^{2}\right)^{-\frac{l+1}{2}} F_{[+]}\left(1+D^{2}\right)^{\frac{l}{2}}\right\| \| 1+D^{2}\right)^{-\frac{l}{2}} \bar{B} \| d v \\
\leq & C_{2}\left(\operatorname{Tr} e^{-\frac{t}{2} D^{2}}\right)^{u}(u t)^{-\frac{l}{2}+1}
\end{aligned}
$$

where we use $F_{[+]}$is a fibrewise first order differential operator and the equality

$$
\sup \left\{(1+x)^{\frac{l}{2}} e^{-\frac{u t x}{2}}\right\}=(u t)^{-\frac{l}{2}} e^{-\frac{l-u t}{2}} .
$$

In the above estimate, we omit the norm of coefficient forms since $B$ is compact. For $J_{1}$ and $J_{2}$ we have similar estimates. For the general $m$, we get

$$
\begin{aligned}
& \|(-u t)^{m} \int_{\triangle_{m}} e^{-v_{0} u t D^{2}} F_{[+]} e^{-v_{1} u t D^{2}} F_{[+]} \cdots e^{-v_{m-1} u t D^{2}} \\
& \cdot F_{[+]} e^{-v_{m} u t D^{2}} \bar{B} d v \|_{u^{-1}} \leq C_{2}\left(\operatorname{Tr} e^{-\frac{t}{2} D^{2}}\right)^{u}(u t)^{-\frac{l}{2}+\frac{m}{2}} .
\end{aligned}
$$


By (4.3) and (4.6), we get (4.2).

LEMMA 4.3. Let $\bar{B}_{1}, \bar{B}_{2}$ be positive order $p$, q fibrewise pseudodifferential operators with form coefficients respectively, then for any $s, t>0,0 \leq u \leq 1$, we have the following estimate:

$$
\left\|\bar{B}_{1} e^{-u s t F} \bar{B}_{2} e^{-(1-u) s t F}\right\|_{s^{-1}} \leq C_{p, q} s^{-\frac{p+q}{2}} t^{-\frac{p+q}{2}}\left(\operatorname{tr}\left[e^{-\frac{t D^{2}}{4}}\right]\right)^{s} .
$$

Proof. Similar to the proof of Lemma 4.2, we have when $t$ is small and $u \geq \frac{1}{2}$,

$$
\left\|\left(1+D^{2}\right)^{-\frac{q}{2}} e^{-\left(u-\frac{1}{2}\right) s t F}\left(1+D^{2}\right)^{\frac{q}{2}}\right\| \leq C_{0} ; \quad\left\|e^{-(1-u) s t F}\right\| \leq C_{1},
$$

where $C_{0}, C_{1}$ are constants which do not depend on $t, u$. When $u \geq \frac{1}{2}$, by Lemma 4.2 and (4.8), we have:

$$
\begin{aligned}
& \left\|\bar{B}_{1} e^{-u s t F} \bar{B}_{2} e^{-(1-u) s t F}\right\|_{s^{-1}} \\
\leq & \left\|\bar{B}_{1}\left(1+D^{2}\right)^{-\frac{p}{2}}\right\|\left\|\left(1+D^{2}\right)^{\frac{p}{2}} e^{-\frac{1}{2} s t F}\left(1+D^{2}\right)^{\frac{q}{2}}\right\|_{s^{-1}} \\
& \cdot\left\|\left(1+D^{2}\right)^{-\frac{q}{2}} e^{-\left(u-\frac{1}{2}\right) s t F}\left(1+D^{2}\right)^{\frac{q}{2}}\right\|\left\|\left(1+D^{2}\right)^{-\frac{q}{2}} \bar{B}_{2}\right\|\left\|e^{-(1-u) s t F}\right\| \\
\leq & C_{p, q} s^{-\frac{p+q}{2}} t^{-\frac{p+q}{2}}\left(\operatorname{tr}\left[e^{-\frac{t D^{2}}{4}}\right]\right)^{s},
\end{aligned}
$$

where we use

$$
\mid\left(1+D^{2}\right)^{\frac{p}{2}} e^{-\frac{1}{2} s t F}\left(1+D^{2}\right)^{\frac{q}{2}}\left\|_{s^{-1}}=\right\| e^{-\frac{1}{2} s t F}\left(1+D^{2}\right)^{\frac{p+q}{2}} \|_{s^{-1}} .
$$

When $u \leq \frac{1}{2}$, by Lemma 4.2 , we have:

$$
\begin{aligned}
& \left\|\bar{B}_{1} e^{-u s t F} \bar{B}_{2} e^{-(1-u) s t F}\right\|_{s^{-1}} \\
\leq & \left\|\bar{B}_{1} e^{-u s t F}\left(1+D^{2}\right)^{-\frac{p}{2}}\right\|\left\|\left(1+D^{2}\right)^{\frac{p}{2}} \bar{B}_{2} e^{-\frac{1}{2} s t F}\right\|_{s^{-1}}\left\|e^{-\left(1-u-\frac{1}{2}\right) s t F}\right\| \\
\leq & C_{p, q} s^{-\frac{p+q}{2}} t^{-\frac{p+q}{2}}\left(\operatorname{tr}\left[e^{-\frac{t D^{2}}{4}}\right]\right)^{s} .
\end{aligned}
$$

By (4.9) and (4.11), we have proved this lemma. Write

Let $\bar{B}$ be a fibrewise operator with form coefficients and $l$ be a positive interger.

$$
\bar{B}^{[l]}=\left[F, \bar{B}^{[l-1]}\right], \bar{B}^{[0]}=\bar{B}
$$

LEMMA 4.4. Let $\bar{B}$ a finite order fibrewise differential operator with form coeffcients, then for any $s>0$, we have:

$$
e^{-s F \bar{B}}=\sum_{l=0}^{N-1} \frac{(-1)^{l}}{l !} s^{l} \bar{B}^{[l]} e^{-s F}+(-1)^{N} s^{N} \bar{B}^{[N]}(s),
$$

where $\bar{B}^{[N]}(s)$ is given by

$$
\bar{B}^{[N]}(s)=\int_{\triangle_{N}} e^{-u_{1} s F} \bar{B}^{[N]} e^{-\left(1-u_{1}\right) s F} d u_{1} d u_{2} \cdots d u_{N} .
$$


Proof. Here we use Lemma 1.9 in $[\mathrm{BeC}]$,

$$
\left[A, e^{-F}\right]=-\int_{0}^{1} e^{-s F}[A, F] e^{-(1-s) F} d s,
$$

then similar to Lemma 3 in $[\mathrm{Fe}]$, we prove this lemma.

For $h_{j} \otimes f_{j}$, we have

$$
\left[\mathcal{B}, h_{j} \otimes f_{j}\right]=d_{B}\left(h_{j} \otimes f_{j}\right) \wedge+h \otimes[D, f] .
$$

Write $T_{j}$ is $d_{B}\left(h_{j} \otimes f_{j}\right) \wedge$ or $h \otimes[D, f], 1 \leq j \leq 2 k$ and $T_{0}=h_{0} \otimes f_{0}$. By Lemma 4.4, we have:

$$
\begin{aligned}
& T_{0} e^{-s_{1} t F} T_{1} e^{-\left(s_{2}-s_{1}\right) t F} T_{2} \cdots e^{-\left(s_{2 k}-s_{2 k-1}\right) t F} T_{2 k} e^{-\left(1-s_{2 k}\right) t F} \\
= & \sum_{\lambda_{1}, \cdots, \lambda_{2 k}=0}^{N-1} \frac{(-1)^{\lambda_{1}+\cdots+\lambda_{2 k}} s_{1}{ }^{\lambda_{1}} \cdots s_{2 k}^{\lambda_{2 k}} t^{\lambda_{1}+\cdots+\lambda_{2 k}}}{\lambda_{1} ! \cdots \lambda_{2 k} !} T_{0}\left[T_{1}\right]^{\left[\lambda_{1}\right]} \cdots\left[T_{2 k}\right]^{\left[\lambda_{2 k}\right]} e^{-t F} \\
& +\sum_{1 \leq q \leq 2 k} \sum_{\lambda_{1}, \cdots, \lambda_{q-1}=0}^{N-1} \frac{(-1)^{\lambda_{1}+\cdots+\lambda_{q-1}+N} s_{1}^{\lambda_{1}} \cdots s_{q-1}^{\lambda_{q-1}} s_{q}^{N} t^{\lambda_{1}+\cdots+\lambda_{q-1}+N}}{\lambda_{1} ! \cdots \lambda_{q-1} !} T_{0}\left[T_{1}\right]^{\left[\lambda_{1}\right]} \\
& \cdots\left[T_{q-1}\right]^{\left[\lambda_{q-1}\right]}\left\{\left[T_{q}\right]^{[N]}\left(s_{q} t\right)\right\} e^{-\left(s_{q+1}-s_{q}\right) t D^{2}} \cdots T_{2 k} e^{-\left(1-s_{2 k}\right) t F} .
\end{aligned}
$$

Since $T_{0}\left[T_{1}\right]^{\left[\lambda_{1}\right]} \cdots\left[T_{q-1}\right]^{\left[\lambda_{q-1}\right]}$ is a $\lambda_{1}+\cdots+\lambda_{q-1}$ order differential operator and by Lemma 4.2 and Lemma 4.3, we get (see pp. 61-62 in [Fe])

$$
\begin{aligned}
& \quad \| \psi_{t} \int_{\Delta_{2 k}} t^{k} \sum_{1 \leq q \leq 2 k} \\
& \quad \times \sum_{\lambda_{1}, \cdots, \lambda_{q-1}=0}^{N-1} \frac{(-1)^{\lambda_{1}+\cdots+\lambda_{q-1}+N} s_{1}^{\lambda_{1}} \cdots s_{q-1}^{\lambda_{q-1}} s_{q}^{N} t^{\lambda_{1}+\cdots+\lambda_{q-1}+N}}{\lambda_{1} ! \cdots \lambda_{q-1} !} T_{0}\left[T_{1}\right]^{\left[\lambda_{1}\right]} \\
& \quad \cdots\left[T_{q-1}\right]^{\left[\lambda_{q-1}\right]}\left\{\left[T_{q}\right]^{[N]}\left(s_{q} t\right)\right\} e^{-\left(s_{q+1}-s_{q}\right) t D^{2}} \cdots T_{2 k} e^{-\left(1-s_{2 k}\right) t F} d v \| \\
& \sim O\left(t^{\frac{2 k+N+\lambda_{1}+\cdots+\lambda_{2 q}-\operatorname{dim} M}{2}}\right) .
\end{aligned}
$$

So we get

Theorem 4.5. (1) if $2 k \leq \operatorname{dim} M$, then

$$
\begin{aligned}
& \mathrm{Ch}_{2 k}\left(\mathcal{B}_{t}\right)(\phi)\left(h_{0} \otimes f_{0}, \cdots, h_{2 k} \otimes f_{2 k}\right) \\
= & \psi_{t} \sum_{\lambda_{1}, \ldots, \lambda_{2 k}=0}^{\operatorname{dim} M-2 k} \frac{(-1)^{\lambda_{1}+\cdots+\lambda_{2 k}}}{\lambda_{1} ! \cdots \lambda_{2 k} !} C t^{|\lambda|+k} \operatorname{Str}\left[\widetilde{\phi} T_{0}\left[T_{1}\right]^{\left[\lambda_{1}\right]} \cdots\left[T_{2 k}\right]^{\left[\lambda_{2 k}\right]} e^{-t F}\right]+O(\sqrt{t}),
\end{aligned}
$$

with the constant

$$
C=\frac{1}{\lambda_{1}+1} \frac{1}{\lambda_{1}+\lambda_{2}+2} \cdots \frac{1}{\lambda_{1}+\cdots+\lambda_{2 k}+2 k} .
$$

(2) if $2 k>\operatorname{dim} M$, then

$$
\mathrm{Ch}_{2 k}\left(\mathcal{B}_{t}\right)(\phi)\left(h_{0} \otimes f_{0}, \cdots, h_{2 k} \otimes f_{2 k}\right)=0 .
$$


Write $P_{k, \lambda}:=T_{0}\left[T_{1}\right]^{\left[\lambda_{1}\right]} \cdots\left[T_{2 k}\right]^{\left[\lambda_{2 k}\right]}$. We will compute

$$
\lim _{t \rightarrow 0^{+}} \psi_{t} t^{|\lambda|+k} \operatorname{Str}\left[\widetilde{\phi} P_{k, \lambda} e^{-t F}\right]
$$

By Lemma 2.18 and Proposition 2.21, we have

$$
\sigma\left(P_{k, \lambda}\right)=T_{0}\left[F_{(2)}, \sigma\left(T_{1}\right)\right]^{\left[\lambda_{1}\right]} \cdots\left[F_{(2)}, \sigma\left(T_{2 k}\right)\right]^{\left[\lambda_{2 k}\right]}+O_{G}(2|\lambda|+2 k-1) .
$$

Direct computations show that

$$
\left[F_{(2)}, \sigma\left(T_{j}\right)\right]=O_{G}(2), \quad O_{G}\left(\left[F_{(2)}, \sigma\left(T_{j}\right)\right]^{\left[\lambda_{j}\right]}\right)<2 \lambda_{j}+1 .
$$

Then when $\left(\lambda_{1}, \cdots, \lambda_{2 k}\right) \neq(0, \cdots, 0)$, then

$$
O_{G}\left(P_{k, \lambda}\right)=O_{G}(2|\lambda|+2 k-1) ; \quad O_{G}\left(P_{k, \lambda}\left(F+\partial_{t}\right)^{-1}\right)=O_{G}(2|\lambda|+2 k-3) .
$$

By Lemma 3.5 and Lemma 3.6 (1), we have

$$
\sigma\left[\psi_{t} I_{P_{k, \lambda}\left(F+\partial_{t}\right)^{-1}}(0, t)\right]^{(a, 0)}=\omega^{\text {odd }} O\left(t^{-|\lambda|-k+\frac{1}{2}}\right)+O\left(t^{-|\lambda|-k+1}\right),
$$

where $\omega^{\text {odd }} \in \Omega^{\text {odd }}(B)$. So in this case,

$$
\lim _{t \rightarrow 0^{+}} \psi_{t} t^{|\lambda|+k} \operatorname{Str}\left[\widetilde{\phi} P_{k, \lambda} e^{-t F}\right]=0 .
$$

When $\left(\lambda_{1}, \cdots, \lambda_{2 k}\right)=(0, \cdots, 0)$. Then $O_{G}\left(T_{0} T_{1} \cdots T_{2 k}\right)=2 k$ and $O_{G}\left(P_{k, \lambda}(F+\right.$ $\left.\left.\partial_{t}\right)^{-1}\right)=O_{G}(2 k-2)$. The model operator of $P_{k, \lambda}\left(F+\partial_{t}\right)^{-1}$ is $T_{0} \sigma\left(T_{1}\right) \cdots \sigma\left(T_{2 k}\right)\left(F_{(2)}+\right.$ $\left.\partial_{t}\right)^{-1}$. By Lemma 3.6(2), we get

$$
\lim _{t \rightarrow 0^{+}} t^{k} \sigma\left[\psi_{t} I_{P_{k, \lambda}\left(F+\partial_{t}\right)^{-1}}(0, t)\right]^{(a, 0)}=T_{0} \sigma\left(T_{1}\right) \cdots \sigma\left(T_{2 k}\right) I_{\left(F_{(2)}+\partial_{t}\right)^{-1}}(0,1)^{(a, 0)} .
$$

By (4.25) and (4.26) and the lemma 9.13 in [PW], we get

THEOREM 4.6. The following equality holds

$$
\begin{aligned}
& \lim _{t \rightarrow 0^{+}} \mathrm{Ch}_{2 k}\left(\mathcal{B}_{t}\right)(\phi)\left(h_{0} \otimes f_{0}, \cdots, h_{2 k} \otimes f_{2 k}\right) \\
= & \frac{1}{(2 k) !}(-i)^{\frac{n}{2}} \sum_{a}(2 \pi)^{-\frac{a}{2}} \int_{M_{a}^{\phi} / B} T_{0} \sigma\left(T_{1}\right) \cdots \sigma\left(T_{2 k}\right) \widehat{A}\left(R^{G^{\phi}}\right) \nu_{\phi}\left(R^{N^{\phi}}\right) .
\end{aligned}
$$

\section{The regularity of the equivariant eta form.}

5.1. The regularity of the equivariant eta invariant. In $[\mathrm{BiF}]$, Bismut and Freed gave a simple proof of the regularity of eta invariants. In [Po2], Ponge gave another proof of the regularity of eta invariants using the method in [Po1]. Zhang proved the regularity of the equivariant eta invariant by the Clifford asymptotics in [Zh3]. In this section, we shall extend the approach of [Po2] to the equivariant setting and prove the regularity of the equivariant eta invariant by this approach. Then we define the equivariant eta form and prove its regularity.

We shall give some notations. Let $X$ be a compact oriented odd dimensional Riemannian manifold without boundary with a fixed spin structure and $S$ be the bundle of spinor on $X$. Denote by $D$ the associated Dirac operator on $H=L^{2}(X ; S)$, the Hilbert space of $L^{2}$-sections of the bundle $X$. Suppose that $\phi$ acts on $X$ by orientation-preserving isometries and $\phi$ has a lift $\widetilde{\phi}: \Gamma(S) \rightarrow \Gamma(S)$ (see [LYZ]), then 
we have $\widetilde{\phi}$ commutes with the Dirac operator and $\widetilde{\phi}$ is a bounded operator. Then the equivariant eta invariant is defined by

$$
\eta_{\phi}(D)=\frac{1}{\sqrt{\pi}} \int_{0}^{\infty} \frac{1}{t^{\frac{1}{2}}} \operatorname{Tr}\left[\widetilde{\phi} D e^{-t D^{2}}\right] d t .
$$

We have

Theorem 5.1 ([Zh3]). As $t \rightarrow 0^{+}$

$$
\operatorname{Tr}\left[\widetilde{\phi} D e^{-t D^{2}}\right] \sim O\left(t^{\frac{1}{2}}\right) .
$$

In order to prove Theorem 5.1, we introduce an auxiliary Grassmann variable $z$ as in $[\mathrm{BiF}]$, i. e. $z^{2}=0$. The auxiliary Grassmann variable $z$ may be considered as one form on the base $S^{1}$ of the fibre bundle $X \times S^{1}$ and set $O_{G}(z)=1$. By the Duhamel principle, we have

$$
\exp \left(-t\left(D^{2}-z D\right)\right)=\exp \left(-t D^{2}\right)+z t D \exp \left(-t D^{2}\right)
$$

Set

$$
h(x)=1+\frac{1}{2} z \sum_{i=1}^{n} x_{i} c\left(e_{i}\right),
$$

where $\left(x_{1}, \cdots, x_{n}\right)$ is the normal coordinates under the parallel frame $e_{1}, \cdots, e_{n}$ and we consider $h$ as $h \chi$ where $\chi$ is a cut function about $\left(x_{1}, \cdots, x_{n}\right)$. By [Zh3], we have

$$
h c\left(e_{i}\right) h^{-1}=c\left(e_{i}\right)+O_{G}(0) ; \quad h\left(D^{2}-z D\right) h^{-1}=D^{2}+z u,
$$

where $O_{G}(u) \leq 0, u$ contains no $z$ and the equality

$$
z t D \exp \left(-t D^{2}\right)(x, y)=h^{-1}(x) \exp \left(-t\left(D^{2}+z u\right)(x, y)\right) h(y)-\exp \left(-t D^{2}\right)(x, y) .
$$

Let

$$
\left(D^{2}+z u\right)^{-1}=D^{-2}-z D^{-2} u D^{-2} .
$$

We may consider $D^{2}+z u$ as the operator with 1 -form coefficients on $X \times S^{1}$. Then the Greiner's approach of the heat kernel asymptotics with form coefficients in Section 2.1 works for $D^{2}+z u$. By

$$
\begin{gathered}
\left(D^{2}+z u+\partial_{t}\right)^{-1}=\left(D^{2}+\partial_{t}\right)^{-1}-z\left(D^{2}+\partial_{t}\right)^{-1} u\left(D^{2}+\partial_{t}\right)^{-1} . \\
K_{\left(D^{2}+z u+\partial_{t}\right)^{-1}}(x, y, t)=K_{\left(D^{2}+\partial_{t}\right)^{-1}}(x, y, t)-z K_{\left(D^{2}+\partial_{t}\right)^{-1} u\left(D^{2}+\partial_{t}\right)^{-1}}(x, y, t) .
\end{gathered}
$$

Then

$$
\begin{aligned}
& \widetilde{\phi}(x) h^{-1}(x) K_{\left(D^{2}+z u+\partial_{t}\right)^{-1}}(x, \phi(x), t) h(\phi(x)) \\
= & \widetilde{\phi}(x) h^{-1}(x) K_{\left(D^{2}+\partial_{t}\right)^{-1}}(x, \phi(x), t) h(\phi(x)) \\
& -z \widetilde{\phi}(x) K_{\left(D^{2}+\partial_{t}\right)^{-1} u\left(D^{2}+\partial_{t}\right)^{-1}}(x, \phi(x), t) .
\end{aligned}
$$


Recall since the dimension $n$ is odd, we have

$$
\operatorname{Tr}\left[c\left(e^{i_{1}}\right) \cdots c\left(e^{i_{k}}\right)\right]=\left\{\begin{array}{cl}
2^{[n / 2]} & \text { if } k=0 \\
0 & \text { if } 0<k<n \\
(-i)^{[n / 2]+1} 2^{[n / 2]} & \text { if } k=n .
\end{array}\right.
$$

Similar to Lemma 3.5, we have:

Lemma 5.2. Let $A \in \mathrm{Cl}^{\text {odd }}(n) \subset \operatorname{End}\left(S_{n}\right)$, then

$$
\begin{aligned}
\operatorname{Tr}[\widetilde{\phi} A]= & (-i)^{\frac{n+1}{2}} 2^{\frac{n-1}{2}} 2^{-\frac{b}{2}} \operatorname{det}^{\frac{1}{2}}\left(1-\phi^{N}\right)|\sigma(A)|^{(a, 0)} \\
& +(-i)^{\frac{n+1}{2}} 2^{\frac{n-1}{2}} \sum_{0 \leq b^{\prime}<b}\left|\sigma(\widetilde{\phi})^{\left(0, b^{\prime}\right)} \sigma(A)^{\left(a, b-b^{\prime}\right)}\right|^{(n)} .
\end{aligned}
$$

We know that Lemma 9.12 in [PW] also holds for the odd dimensional manifold $X$ and when $a$ is odd. We know that $\left(D^{2}+\partial_{t}\right)^{-1} u\left(D^{2}+\partial_{t}\right)^{-1}$ has the Getzler order -4 and odd Clifford elements. By Lemma 8.1 and Lemma 9.12 in [PW] and Lemma 5.2 for $\left(D^{2}+\partial_{t}\right)^{-1} u\left(D^{2}+\partial_{t}\right)^{-1}$ and $j \geq a$, so

$$
\int_{X} \operatorname{Tr}\left[\widetilde{\phi} K_{\left(D^{2}+\partial_{t}\right)^{-1} u\left(D^{2}+\partial_{t}\right)^{-1}}(x, \phi(x), t)\right] d x=O\left(t^{\frac{3}{2}}\right) .
$$

By equalities (5.10) and (5.13), in order to prove Theorem 5.1, we need to prove

$$
\widetilde{\phi}(x) h^{-1}(x) K_{\left(D^{2}+\partial_{t}\right)^{-1}}(x, \phi(x), t) h(\phi(x))=\widetilde{\phi}(x) K_{\left(D^{2}+\partial_{t}\right)^{-1}}(x, \phi(x), t)+O\left(t^{\frac{3}{2}}\right) .
$$

This comes from the equality

$$
h^{-1}\left(x^{\prime}, v\right) c\left(e_{i}\right) h\left(x^{\prime}, \phi^{\prime}(x) v\right)=c\left(e_{i}\right)+\frac{1}{2} z c\left(e_{i}\right) \sum_{j=a+1}^{n}\left[\left(\phi^{\prime}(x)-1\right) v\right]_{j} c\left(e_{j}\right)-z x_{i},
$$

and $\widetilde{\phi}$ only contains the Clifford elements $c\left(e_{a+1}\right), \cdots, c\left(e_{n}\right)$.

5.2. The regularity of the equivariant eta form. In this section, the fundamental setup is the same as Section 2.1. But we assume the dimension $n$ of the fibre to be odd. Let $\phi$ be an isometry which acts fiberwise on $M$. We will consider that $\phi$ acts as identity on $B$. Let $\operatorname{Tr}^{\text {even }}$ be the trace on the coefficients of even forms on $B$. We assume that $\operatorname{ker} D$ is a complex vector bundle. Then the equivariant eta form is defined by

$$
\widehat{\eta}(\phi):=\int_{0}^{\infty} \operatorname{Tr}^{\mathrm{even}}\left[\widetilde{\phi} \frac{d \mathcal{B}_{t}}{d t} e^{-\mathcal{B}_{t}^{2}}\right] d t
$$

Let $T$ be the torsion tensor of $\nabla^{\oplus}$ and $c(T)=\sum_{1 \leq \alpha<\beta \leq m} d y_{\alpha} d y_{\beta} c\left(T\left(\frac{\partial}{\partial y_{\alpha}}, \frac{\partial}{\partial y_{\beta}}\right)\right)$. Then

$$
\widehat{\eta}(\phi)=\int_{0}^{\infty} \frac{1}{2 \sqrt{t}} \operatorname{Tr}^{\text {even }}\left[\psi_{t} \widetilde{\phi}\left(D+\frac{c(T)}{4}\right) e^{-t F}\right] d t
$$


Lemma 5.3. We assume that $\operatorname{ker} D$ is a complex vector bundle. As $t \rightarrow+\infty$, we have

$$
\frac{1}{2 \sqrt{t}} \operatorname{Tr}^{\text {even }}\left[\psi_{t} \widetilde{\phi}\left(D+\frac{c(T)}{4}\right) e^{-t F}\right] \sim O\left(t^{-\frac{3}{2}}\right)
$$

Proof. Since $\widetilde{\phi}$ is a bounded operator, so the proof of Lemma 5.3 is the same as the proof of Theorem 9.7 in [BGV].

Let $e_{1}(x), \cdots e_{n}(x)$ denote the orthonormal frame of $T Z$. If $A(Y)$ is any 0 order operator depending linearly on $Y \in \Gamma(M, T M)$, we define the operator $\left(\nabla_{e_{i}}+A\left(e_{i}\right)\right)^{2}$ as follows

$$
\left(\nabla_{e_{i}}+A\left(e_{i}\right)\right)^{2}=\sum_{1}^{n}\left(\nabla_{e_{i}(x)}+A\left(e_{i}(x)\right)\right)^{2}-\nabla_{\sum_{j} \nabla_{e_{j}} e_{j}}-A\left(\sum_{j} \nabla_{e_{j}} e_{j}\right) .
$$

Then by [Bi2], we have

$$
F=-\left(\nabla_{e_{i}}^{T Z}+\frac{1}{2}<S\left(e_{i}\right) e_{j}, f_{\alpha}>e_{j} d y_{\alpha}+\frac{1}{4}<S\left(e_{i}\right) f_{\alpha}, f_{\beta}>d y_{\alpha} d y_{\beta}\right)^{2}+\frac{r}{4}
$$

Set

$$
h(x)=1+\frac{1}{2} d t \sum_{i=1}^{n} x_{i} e_{i}
$$

Then we have

$$
\begin{gathered}
h e_{i} h^{-1}=e_{i}+d t L_{1} ; \quad h\left(\frac{1}{2} e_{i} d t\right) h^{-1}=\frac{1}{2} e_{i} d t, \\
h \nabla_{e_{i}}^{G} h^{-1}=\nabla_{e_{i}}^{G}-\frac{1}{2} d t e_{i}+d t L_{2}, \\
h\left(\frac{1}{2}<S\left(e_{i}\right) e_{j}, f_{\alpha}>e_{j} d y_{\alpha}\right) h^{-1}=\frac{1}{2}<S\left(e_{i}\right) e_{j}, f_{\alpha}>e_{j} d y_{\alpha}+d t L_{3}, \\
h\left(\frac{1}{4}<S\left(e_{i}\right) f_{\alpha}, f_{\beta}>d y_{\alpha} d y_{\beta}\right) h^{-1}=\frac{1}{4}<S\left(e_{i}\right) f_{\alpha}, f_{\beta}>d y_{\alpha} d y_{\beta},
\end{gathered}
$$

where $L_{1}, L_{2}, L_{3} \in O_{G}(-1)$. By Proposition 2.10 in [BeGS], we have

$$
\begin{aligned}
F+d t\left(D+\frac{c(T)}{4}\right)= & -\left(\nabla_{e_{i}}^{T Z}+\frac{1}{2}\left\langle S\left(e_{i}\right) e_{j}, f_{\alpha}\right\rangle e_{j} d y_{\alpha}\right. \\
& \left.+\frac{1}{4}\left\langle S\left(e_{i}\right) f_{\alpha}, f_{\beta}\right\rangle d y_{\alpha} d y_{\beta}-\frac{1}{2} e_{i} d t\right)^{2}+\frac{r}{4},
\end{aligned}
$$

By (5.24)-(5.28), we have

$$
h\left[F+d t\left(D+\frac{c(T)}{4}\right)\right] h^{-1}=F+d t u,
$$


where $O_{G}(u) \leq 0$. By the Duhamel principle, we can get

$$
\begin{aligned}
& t d t \operatorname{Tr}^{\operatorname{even}}\left[\widetilde{\phi}\left(D+\frac{c(T)}{4}\right) \exp (-t F)\right] \\
= & \operatorname{Tr}^{\operatorname{even}}[\widetilde{\phi} \exp (-t F)]-\operatorname{Tr}^{\operatorname{even}}\left[\widetilde{\phi} h^{-1} \exp (-t(F+d t u)) h\right] .
\end{aligned}
$$

Then

$$
\begin{aligned}
& t \frac{d t}{\sqrt{t}} \operatorname{Tr}^{\operatorname{even}}\left[\widetilde{\phi}\left(D+\frac{c(T)}{4 t}\right) \psi_{t} \exp (-t F)\right] \\
= & \operatorname{Tr}^{\operatorname{even}}\left[\widetilde{\phi} \psi_{t} \exp (-t F)\right]-\operatorname{Tr}^{\operatorname{even}}\left[\widetilde{\phi} h^{-1} \psi_{t} \exp (-t(F+d t u)) h\right] .
\end{aligned}
$$

We know that Lemma 3.6 is still correct. When we take $\operatorname{Tr}^{\text {even }}$, the terms having coefficients in $\Omega^{\text {odd }}$ vanish. Similar to Section 5.1 , we can get

TheOrem 5.4. As $t \rightarrow 0^{+}$, we have

$$
\operatorname{Tr}^{\text {even }}\left[\psi_{t} \widetilde{\phi}\left(D+\frac{c(T)}{4}\right) e^{-t F}\right] \sim O\left(t^{\frac{1}{2}}\right) .
$$

By Lemma 5.3 and Theorem 5.4, the equivariant eta form is well defined. When $n$ is even, we define

$$
\widehat{\eta}(\phi):=\int_{0}^{\infty} \operatorname{Str}\left[\widetilde{\phi} \frac{d \mathcal{B}_{t}}{d t} e^{-\mathcal{B}_{t}^{2}}\right] d t .
$$

Similarly, we may prove the regularity in this case.

Acknowledgement. This project was supported by NSFC No.11271062 and NCET-13-0721. The author would like to thank Profs. Weiping Zhang and Huitao Feng for introducing index theory to him. The author would like to thank Prof. Weiping Zhang and Dr. Hang Wang for helpful discussions and comments. The author would like to thank referees for careful reading and helpful comments.

\section{REFERENCES}

[APS] M. F. Atiyah, V. K. Patodi, And I. M. Singer, Spectral asymmetry and Riemannian geometry, I. Math. Proc. Cambridge Philos. Soc., 77 (1975), pp. 43-69.

[AS] M. F. Atiyah And I. M. Singer, The index of elliptic operators IV, Annals of Math., 93 (1971), pp. 119-138.

[Az1] F. Azmi, The equivariant Dirac cyclic cocycle, Rocky Mountain J. Math., 30 (2000), pp. 1171-1206.

[Az2] F. Azmi, The Chern-Connes character formula for families of Dirac operators, Publ. Math. Debrecen, 61 (2002), pp. 1-27.

[Az3] F. Azmi, Equivariant bivariant cyclic theory and equivariant Chern-Connes character, Rocky Mountain J. Math., 34:2 (2004), pp. 391-412.

[BeGS] R. Beals, P. Greiner, and N. Stanton, The heat equation on a CR manifold, J. Differential Geom., 20 (1984), pp. 343-387.

[BeC] M. Benameur And A. CARey, Higher spectral flow and an entire bivariant JLO cocycle, J. K-Theory, 11:1(2013), pp. 183-232.

[BGV] N. Berline, E. Getzler, And M. Vergne, Heat kernels and Dirac operators, SpringerVerlag, Berlin, 1992.

[BV1] N. Berline And M. Vergne, A proof of Bismut local index theorem for a family of Dirac operators, Topology, 26:4 (1987), pp. 435-463.

[BV2] N. Berline And M. Vergne, A computation of the equivariant index of the Dirac operators, Bull. Soc. Math. Prance, 113 (1985), pp. 305-345. 
[Bi1] J.-M. Bismut, The Atiyah-Singer theorems: A probabilistic approach, J. Func. Anal., 57 (1984), pp. 56-99.

[Bi2] J.-M. Bismut, The Atiyah-Singer index theorem for families of Dirac operators: Two heat equation proofs, Invent. Math., 83 (1986), pp. 91-151.

[Bi3] J.-M. Bismut, Equivariant immersions and Quillen metrics, J. Diff. Geom., 41 (1995), pp. 53-159.

[BC1] J.-M. Bismut And J. CheEger, Families index for manifolds with boundary, superconnections, and cones. I. Families of manifolds with boundary and Dirac operators, J. Funct. Anal., 89:2 (1990), pp. 313-363.

[BC2] J.-M. Bismut And J. Cheeger, Families index for manifolds with boundary, superconnections and cones. II. The Chern character, J. Funct. Anal., 90:2 (1990), pp. 306-354.

[BiF] J.-M. Bismut And D. Freed, The analysis of elliptic families. II. Dirac operators, eta invariants, and the holonomy theorem, Comm. Math. Phys., 107:1 (1986), pp. 103163.

[BiGS] J.-M. Bismut, H. Gillet, And C. Soulé, Analytic torsion and holomorphic determinant bundles. I. Bott-Chern forms and analytic torsion, Comm. Math. Phys., 115:1 (1988), pp. $49-78$.

[BlF] J. BlOCK AND J. Fox, Asymptotic pseudodifferential operators and index theory, Contemp. Math., 105 (1990), pp. 1-32.

[CH] S. ChERN AND X. Hu, Equivariant Chern character for the invariant Dirac operators, Michigan Math. J., 44 (1997), pp. 451-473.

[Co] A. Connes, Entire cyclic cohomology of Banach algebras and the character of $\theta$-summable Fredholm module, K-Theory, 1 (1988), pp. 519-548.

[CM1] A. Connes And H. Moscovici, Cyclic cohomology, the Novikov conjecture and hyperbolic groups, Topology, 29:3 (1990), pp. 345-388.

[CM2] A. Connes And H. Moscovici, Transgression and Chern character of finite dimensional K-cycles, Commun. Math. Phys., 155 (1993), pp. 103-122.

[CFKS] H. L. Cycon, R. G. Froese, W. Kirsch, And B. Simon, Schrodinger Operators with Application to Quantum Mechanics and Global Geometry, Springer-Verlag, Texts and Monographs in Physics, 1987.

[Do1] H. Donnelly, Local index theorem for families, Michigan Math. J., 35:1 (1988), pp. 11-20.

[Do2] H. Donnelly, Eta invariants for G-spaces, Indiana Univ. Math. J., 27:6 (1978), pp. 889918.

[Fe] H. FEnG, A note on the noncommutative Chern character (in Chinese), Acta Math. Sinica, 46 (2003), pp. 57-64.

[Ge1] E. GetZler, PseudodifferentiaI operators on supermanifolds and the Atiyah-Singer index theorem, Commun. Math. Phys., 92 (1983), pp. 163-178.

[Ge2] E. Getzler, A short proof of the local Atiyah-Singer index theorem, Topology, 25 (1986), pp. 111-117.

[Gr] P. Greiner, An asymptotic expansion for the heat equation, Arch. Rational Mech. Anal., 41 (1971), pp. 163-218.

[JLO] A. Jaffe, A. Lesniewski, And K. Osterwalder, Quantum K-theory: The Chern character, Comm. Math. Phys., 118 (1988), pp. 1-14.

[LYZ] J. D. Lafferty, Y. L. Yu, And W. P. Zhang, A direct geometric proof of Lefschetz fixed point formulas, Trans. AMS., 329 (1992), pp. 571-583.

[LM] K. Liu AND X. MA, On family rigidity theorems. I, Duke Math. J., 102:3 (2000), pp. 451474.

[Lo] J. LotT, Superconnections and higher index theory, Geom. Func. Anal., 2 (1992), pp. 421454 .

[MS] H. P. Mckean And I. M. Singer, Curvature and the eigenvalue of the Laplacian, J. Diff. Geom., I (1967), pp. 43-69.

[Pa] V. K. PAtodi, Curvatures and the eigenforms of the Laplace operator, J. Diff. Geom., 5 (1971), pp. 238-249.

[Po1] R. POnge, A new short proof of the local index formula and some of its applications, Comm. Math. Phys., 241 (2003), pp. 215-234.

[Po2] R. Ponge, A new proof of the local regularity of the eta invariant of a Dirac operator, J. Geom. Phys., 56:9 (2006), pp. 1654-1665.

[PW] R. Ponge And H. Wang, Noncommutative geometry, conformal geometry, and the local equivariant index theorem, arXiv:1411.3703.

[Qu] D. Quillen, Superconnections and the Chern character, Topology, 24 (1985), pp. 89-95.

[Si] B. Simon, Trace ideals and their applications, London Math. Soc., Lecture Note 35, 1979.

[Ta] M. E. TAYlor, Pseudodifferential operators, Princeton Mathematical Series, 34. Princeton 
University Press, Princeton, N.J., 1981.

[Wu $\quad \mathrm{F} . \mathrm{Wu}, A$ bivariant Chern-Connes character and the higher $\Gamma$-index theorem, K-Theory, 11:1 (1997), pp. 35-82.

[Yu] Y. YU, Local index theorem for Dirac operators, Acta. Math. Sinica., 3:2 (1987), pp. 152169.

[Zh1] W. ZhAng, Local Atiyah-Singer index theorem for families of Dirac operators, Differential geometry and topology, Lecture Notes in Math., 1369, Springer, Berlin, 1989, pp. 351366 .

[Zh2] W. Zhang, The Chern-Connes character and the local family index theorem, preprint.

[Zh3] W. Zhang, A note on equivariant eta invariants, Proc. AMS, 108:4 (1990), pp. 1121-1129. 
